\title{
On Purely Mechanical Simple Kinematic Internal Constraints
}

\author{
Adam Zdunek ${ }^{1}$
}

Received: 30 May 2018 / Published online: 2 September 2019

(C) The Author(s) 2019

\begin{abstract}
The classic purely mechanical approach to materials with simple kinematic internal constraints is supplemented. A right Cauchy-Green tensor which locally represents the kinematically admissible restricted domain of a finite hyperelastic stress response function is constructed explicitly. It satisfies all imposed constraints identically. It is obtained by a procedure which annihilates the banned modes of deformation in the actual placement. A unique direct sum based stress decomposition is obtained. Further, a procedure is provided, to seamlessly relax constraints, or reversibly, that allows constraints to smoothly develop under loading starting from an unconstrained description. The involved relaxation of internal constraints is briefly illustrated herein. References to published full feathered applications are given where the method is used and verified in finite element form.
\end{abstract}

Keywords Internal constraints · Incompressible $\cdot$ Inextensible $\cdot$ Angle preserving · Constrained convected metric

Mathematics Subject Classification (2000) 74A05 · 74A20 · 74B20 · 74S05

\section{Introduction}

The standard reference to the classic purely mechanic theory of simple kinematic constraints is Truesdell and Noll [1, Sect. 30]. It covers the historical aspects and provides an axiomatic presentation of theory with examples to incompressibility and simple inextensibility. It defines the mechanical reactions to imposed purely kinematic constraints as the energy conjugate stress, that is workless in all deformations satisfying the constraint. Further, the classic theory defines the constitutively determinate work performing stress, called the extra stress, as the difference between the total stress and the workless, constitutively indeterminate, stress. Thus, it introduces an additive decomposition of the total stress. Relying on these definitions, the so-called Principle of material determinism for simple materials subject to

A. Zdunek

adam.zdunek@foi.se

1 Swedish Defence Research Agency, FOI, 16490 Stockholm, Sweden 
internal constraints is laid down. Notably, it leaves the mechanical interpretation of the introduced Lagrange multipliers undetermined.

Later, the so-called constraint manifold theory by Podio-Guidugli [3], Gurtin and PodioGuidugli [5], Podio-Guidugli and Vianello [4] and recently Vianello [6], and by Carlson et al. [7], to mention a few, updated the classic theory using geometric-algebraic analogies from linear-algebra. The additive stress decomposition emerges from spanning the workless stress on the normals to the set of constraints. The work-performing stress is isolated as the orthogonal complement, spanned by the tangent set.

The normalisation condition removing the indeterminate part from the extra stress, which makes the stress decomposition unique, is only mentioned as an option in [1, Sect. 30]. It is invoked firmly in the constraint manifold theory (see, e.g., [7]), where orthogonality is assumed between the workless and the work-performing stresses. The constraint manifold theory concludes making the direct sum based stress decomposition unique by constructing the orthogonal projection to the set of constraint normals. It is concluded that the normalisation condition as presented in [1, Sect. 30] and in [7] is an auxiliary assumption to make the stress decomposition unique.

According to Steigmann [11] the subject of materials with internal constraints is not especially well treated in text and monographs. The necessity and usefulness of a normalisation condition, introduced in [1, Sect. 30], has been questioned, see for example Bertram and Glüge [9]. Negahban [10] discusses different assumed stress decompositions and normalisations. Normalised work performing stresses are more seldom used in analytic works. See, for example, the presentation of the classic families of controllable non-homogeneous solutions of arbitrary isotropic incompressible materials in Truesdell and Noll [1, Sects. 56 and 57]. In computational mechanics, on the other hand, the advantage of using the normalised deviatoric description of the work-performing stresses in nearly incompressible hyperelasticity was early recognised by Simo, Taylor and Pister [14] and is firmly established.

This contribution is confined to isothermal conditions. The thermodynamic considerations are consequently reduced to the Clausius-Planck form of the second law. The appearing stress reactions herein are constitutively indeterminate, spatial scalar fields. Interesting phenomena per se of type ageing, damage, growth and remodelling are out of the scope. Extensions to thermoelasticity are considered in, $[5,8,10]$. For viscous Newtonian fluids, the dissipation in the presence of an incompressibility constraint is accounted for both mechanically and thermally by the set of Navier-Stokes equations which have a very rich literature. Further, modern elasto-plasticity is based on hyperelasticity while requesting the dissipative plastic flow to be isochoric, governed by the second law of thermodynamics, see Simo and Hughes [24]. Finally, Bertram and Glüge [9] propose extensions to second order gradient materials with internal constraints and discuss additional considerations bringing in thermomechanics. It is concluded that the fundamental aspects of internal constraints, may very well be introduced for (hyper-)elasticity and later be elaborated on for other material classes.

Further, this contribution has application in computational mechanics. There, kinematic internal constraints are part of the strong statement of the boundary value problem, in a Dirichlet sense. That is, the constraint manifold restricts the set of admissible transplacements, also called the set of admissible trial solutions. Its tangent set is known as the set of admissible variations/test functions. Consequently, it is restricted by the first variations of the constraints, i.e., with the tangent set to the constraint manifold.

Putting it all together, this contribution presents the unified theoretical development published as verified finite element implementations [27, 29, 31] which extends the classic theory of kinematic internal constraints, [1, Sect. 30] and [3-5, 7]. The claimed extensions concern, governing theory and procedures for, 
$\left(\mathscr{C}_{1}\right)$ modification of an actual Cauchy-Green tensor to become an element of the constraint manifold, and for,

$\left(\mathscr{C}_{2}\right)$ relaxing constraints ${ }^{1}$ by turning them into variable substitutions, making the material 'nearly' constrained, and finally by,

$\left(\mathscr{C}_{3}\right)$ indicating the impact of these two contributions on the solutions of boundary value problems in computational solid mechanics.

Using the modified Cauchy-Green tensor as an argument of the hyperelastic stored energy function confines its domain to the actual constrained situation. ${ }^{2}$ Using the ClausiusPlanck form of the second law of thermodynamics (for isothermal processes) and employing the famous Coleman Noll procedure [2] induces automatically the proper direct sum decomposition of the stress into a workless reactive part and a work performing active part. ${ }^{3}$ Turning constraint equations into variable substitutions $\left(\mathscr{C}_{2}\right)$ allows a seamless description from unconstrained to fully constrained and reverse under loading for example. Finally, contribution $\left(\mathscr{C}_{3}\right)$ bridges material mechanics and computational mechanics.

Incompressibility, inextensibility and preservation of the angle between two material elements is considered here. Combinations of them are considered as example applications. Put in other words, $\left(\mathscr{C}_{1}\right)$ allows to state an internally constrained finite hyperelastic (boundary value) problem in an inner product vector space that preserves volume, length or angle, simply or in combination. The construction of the constrained right Cauchy-Green stretch tensor is performed by a rank-one update procedure annihilating one target banned mode at the time in the Eulerian convected metric tensor representing the actual transplacement, in the sense of Green an Zerna [15]. In other words, the annihilation is done point-wise by composition in the local material fibre triad. The result is pulled back to the reference placement where the constrained right Cauchy-Green stretch tensor is the Lagrangian form of the Eulerian convected metric tensor. It should not be confused with the augmenting internal variable representing physical metric tensor introduced by Valanis and Valanis and Panoskaltsis $[25,26]$. The right Cauchy-Green tensor and the convected Lagrangian metric tensor are equivalent designations used interchangeably.

Following the fibre deformation in terms of convected local fibre triads is a key ingredient providing maximum possible direct insight into a phenomenologic type of description, [16]. It takes advantage of given preferred directions. The verification of the finite element realisation of the stiffening two-fibre family reinforcement (Example 2 herein), can be found in the recently published work [31]. To my best knowledge, this work is the first of its kind.

This contribution is concluded in that spirit by briefly presenting the option $\left(\mathscr{C}_{2}\right)$ to relax internal constraints keeping ${ }^{4}$ the direct sum decomposition of the stress used in the fully constrained case. Tested and verified finite element implementations which can be interpreted in terms of the so-called generalised metric approach are available. The 3-field, displacement, dilatation and pressure formulation by Simo, Taylor and Pister [14, (1985)] for near incompressibility is the first to be mentioned. The following formulations [27-30] for near incompressibility and/or strongly transversely isotropic finite hyperelasticity are further realizations.

The outline of the remainder of this contribution is: The notation used is given in a short paragraph in the Appendix A. A condensed exposition of the kinematics used is given in

\footnotetext{
${ }^{1}$ Called extrapolating off the manifold by Carlson et al. [7].

${ }^{2}$ For example, the unimodular Cauchy-Green tensor for incompressibility.

${ }^{3}$ For example, decomposition in a spherical and a traceless part for incompressibility.

${ }^{4}$ Transforming the Lagrange multiplier to a constitutively determinate stress response function.
} 
Sect. 2. The kinematic aspects of simple internal constraints are gathered in Sect. 3. The procedure to construct the constrained right Cauchy-Green tensor is presented in Sect. 3.2, $\left(\mathscr{C}_{1}\right)$. The constitutive aspects of simple internal constraints are illustrated for finite hyperelasticity in Sect. 4. The relaxation of internal constraints, in other words, the procedure to connect with the related unconstrained material is briefly presented in Sect. 5. The claimed generalisation of the classic theory is discussed in Sect. 6. The note is summarised and concluded in Sect. 7. Small examples are used to illustrate concepts and show applications throughout the note. Finite element applications are found in [27-31].

\section{Kinematics}

Adopting the continuum mechanics view, let $\kappa_{t}: \mathcal{B} \rightarrow \mathscr{E}$ be a smooth time-dependent embedding of a material body $\mathcal{B}$ into the differentiable Euclidean point manifold $\mathscr{E}$. The translation space at instant $t \geq 0$ associated with $\mathscr{E}$ is denoted $\mathscr{U}_{t}$. The placement of, or region occupied by, the material body at time $t$ is the image $\mathscr{P}_{t}:=\kappa_{t}(\mathcal{B})$. The position of a material particle $X \in \mathcal{B}$ is given by $\boldsymbol{x}_{t}:=\kappa_{t}(X) \in \mathscr{P}_{t}$. The placement $\mathscr{P}_{0}:=\kappa_{0}(\mathcal{B})$ at the instant $t=0$ is used as a reference. The reference position is denoted $X:=\kappa_{0}(X)$. The embedding is invertible, for $\boldsymbol{X} \in \mathscr{P}_{0}, X=\kappa_{0}^{-1}(X) \in \mathcal{B}$. The abbreviated notation $\boldsymbol{x}:=\kappa_{t}(X)$ is used for the actual position and the actual placement is denoted $\mathscr{P}=\kappa_{t}(\mathcal{B})$. The composed point mapping,

$$
\varphi(X, t):=\kappa_{t} \circ \kappa_{0}^{-1}: \mathscr{P}_{0} \mapsto \mathscr{P},
$$

defines a common referential description of the deformation of a material body used in continuum mechanics. It is assumed to be bijective and continuously differentiable with respect to the time parameter $t$ as many times as needed. The tangent map is the linear transformation $\mathbf{F}:=\operatorname{Grad} \varphi: T_{X} \mathscr{P}_{0} \rightarrow T_{x} \mathscr{P}$.

Parametrisation It is henceforth assumed that a locally bijective and smooth parametrization $\xi^{A}(\boldsymbol{X})$ around $\boldsymbol{X} \in \mathscr{P}_{0}$ is given, or that a material curve can be reconstructed from a given distinct unit tangent field $\hat{\boldsymbol{M}}(\boldsymbol{X})$ in $\mathscr{P}_{0}$, called a preferred direction in the phenomenologic theory of anisotropic solids, see Spencer [16], Boehler [17] and Zhang and Rychlewski [18]. A convective local parameterization $\xi^{a}(\boldsymbol{x})$ around $\boldsymbol{x} \in \mathscr{P}$ is chosen, cf. Green and Zerna [15]. Then the natural tangent base vectors $\left\{\boldsymbol{G}_{A}\right\}$ and $\left\{\boldsymbol{g}_{a}\right\}$ on $T_{\boldsymbol{X}} \mathscr{P}_{0}$ and $T_{\boldsymbol{x}} \mathscr{P}$ are defined in a standard fashion by,

$$
\boldsymbol{G}_{A}:=\frac{\partial \boldsymbol{X}(\boldsymbol{\xi})}{\partial \xi^{A}} \quad \text { and } \quad \boldsymbol{g}_{a}:=\frac{\partial \boldsymbol{x}(\boldsymbol{\xi})}{\partial \xi^{a}}
$$

At least one of these base vectors will correspond to a preferred direction, $\hat{\boldsymbol{M}}$ in the presence of an anisotropic constraint. Transverse isotropy caused by a simple inextensibility involves a single preferred direction. The local convected triad is completed by constructing two perpendicular base vectors in the isotropic plane. Local orthotropy, caused by two simple inextensibilities, involves two distinct, in general oblique, preferred directions. The third base vector is constructed using the vector cross product. For isotropy the preferred local triad is constructed to be orthogonal. An initially isotropic solid may in principle develop inextensibility under load. Arterial soft tissue is known to develop Fung-like exponential stiffening. 
The co-vector bases on the dual spaces $T_{\boldsymbol{X}}^{*} \mathscr{P}_{0}$ and $T_{\boldsymbol{x}}^{*} \mathscr{P}$ are denoted $\left\{\boldsymbol{G}^{A}\right\}$ and $\left\{\boldsymbol{g}_{\sim}^{a}\right\}$ respectively. They are determined with the aid of the duality pairing as follows,

$$
\left\langle\underline{\sim}^{A}, \boldsymbol{G}_{B}\right\rangle_{T_{\boldsymbol{X}} \mathscr{P}_{0}}=\delta_{B}^{A} \quad \text { and } \quad\left\langle{\underset{\sim}{\boldsymbol{g}}}^{a}, \boldsymbol{g}_{b}\right\rangle_{T_{\boldsymbol{x}} \mathscr{P}}=\delta_{b}^{a},
$$

where $\delta_{B}^{A}$ and $\delta_{b}^{a}$ are Kronecker delta symbols. The metric tensors on the referential and actual tangent spaces are defined by,

$$
\left\{\begin{array} { l } 
{ \mathbf { G } : = G _ { A B } \boldsymbol { G } ^ { A } \otimes \underline { \boldsymbol { G } } ^ { B } , } \\
{ \mathbf { G } ^ { * } = \mathbf { G } , } \\
{ G : = \operatorname { d e t } [ G _ { A B } ] > 0 , }
\end{array} \quad \text { and } \quad \left\{\begin{array}{l}
\mathbf{g}:=g_{a b}{\underset{\sim}{\mathbf{g}}}^{a} \otimes{\underset{\sim}{\mathbf{g}}}^{b}, \\
\mathbf{g}^{*}=\mathbf{g}, \\
g:=\operatorname{det}\left[g_{a b}\right]>0 .
\end{array}\right.\right.
$$

They are linear maps that are assumed to be symmetric and positive definite ${ }^{5}$ by definition, i.e., $\mathbf{G} \in \operatorname{Sym}^{+}\left(T_{\boldsymbol{X}}^{*} \mathscr{P}_{0} \otimes T_{\boldsymbol{X}}^{*} \mathscr{P}_{0}\right)$ and $\mathbf{g} \in \operatorname{Sym}^{+}\left(T_{\varphi(\boldsymbol{X})}^{*} \mathscr{P} \otimes T_{\varphi(\boldsymbol{X})}^{*} \mathscr{P}\right)$, respectively. Using the convected parametrisation the deformation gradient becomes, $\mathbf{F}=\delta_{{ }^{a}}^{a} \boldsymbol{g}_{a} \otimes \boldsymbol{G}^{A}$. Summing up, the deformation gradient $\mathbf{F}$, its adjoint $\mathbf{F}^{*}$ and their inverses $\mathbf{F}^{-1}$ and $\mathbf{F}^{-*}$ take the forms:

$$
\left.\begin{array}{ll}
\mathbf{F}=\delta_{\cdot A}^{a} \boldsymbol{g}_{a} \otimes{\underset{\sim}{G}}^{A}, & \mathbf{F}^{-1}=\delta_{\cdot a}^{A} \boldsymbol{G}_{A} \otimes{\underset{\sim}{\boldsymbol{g}}}^{a}, \\
\mathbf{F}^{*}=\delta_{\cdot A}^{a} \underline{G}^{A} \otimes \boldsymbol{g}_{a}, & \mathbf{F}^{-*}=\delta_{\cdot a}^{A}{\underset{\sim}{\boldsymbol{g}}}^{a} \otimes \boldsymbol{G}_{A} \cdot
\end{array}\right\}
$$

The basis vectors $\boldsymbol{G}_{A}$ and $\boldsymbol{g}_{a}$ henceforth represent tangents to material curves at $\boldsymbol{X} \in \mathscr{P}_{0}$ and at $\varphi(X, t) \in \mathscr{P}$. In case of anisotropy one or more of these represent convected preferred directions.

In view of (2.4a), the tangent mapping $\mathbf{F}: \boldsymbol{G}_{A} \mapsto \boldsymbol{g}_{a}$ is written;

$$
\hat{\boldsymbol{g}}_{a}=\lambda_{a}^{-1} \mathbf{F} \boldsymbol{G}_{A}, \quad\left\|\hat{\boldsymbol{g}}_{a}\right\|=1 \quad \text { and } \quad \lambda_{a}:=\sqrt{\left\langle\mathbf{g F} \boldsymbol{G}_{A}, \mathbf{F} \boldsymbol{G}_{A}\right\rangle}=\sqrt{\left\langle\mathbf{F}^{*} \mathbf{g F} \boldsymbol{G}_{A}, \boldsymbol{G}_{A}\right\rangle},
$$

where the so-called convected metric tensor $\mathbf{g}=\mathbf{g}(\boldsymbol{\varphi})$ has the explicit form:

$$
\mathbf{g}=\left[\begin{array}{ccc}
\lambda_{1}^{2} & \alpha_{12} \lambda_{1} \lambda_{2} & \alpha_{13} \lambda_{1} \lambda_{3} \\
\bullet & \lambda_{2}^{2} & \alpha_{23} \lambda_{2} \lambda_{3} \\
\bullet & \bullet & \lambda_{3}^{2}
\end{array}\right]_{a b} \underset{\sim}{\boldsymbol{g}^{a}} \otimes \underset{\sim}{\operatorname{g}} b,\left.\quad \mathbf{g}(\boldsymbol{\varphi})\right|_{\varphi=X}=\mathbf{G}(\boldsymbol{X}),
$$

where $\lambda_{a}^{2}:=\left\|\boldsymbol{g}_{a}\right\|^{2}$ are stretches and $\alpha_{a b}:=\lambda_{a}^{-1} \lambda_{b}^{-1} \boldsymbol{g}_{a} \cdot \boldsymbol{g}_{b}$ are the direction cosines, i.e., the shears, between the material line elements $\boldsymbol{g}_{a}$ and $\boldsymbol{g}_{b}$ for $a, b=1,2,3$ with $b>a$, in $T_{\varphi(X)} \mathscr{P}$, respectively. Further, is noted that the referential value of convected metric tensor $(2.6)_{2}$ has to be provided. It is constructed exploiting any given preferred direction as outlined in the paragraph Parametrisation above.

Take for example strong transverse isotropy, caused by an inextensibility constraint, one of the stretches in (2.6) measures the length ratio in the associated preferred direction. Likewise, a direction cosine will measure the actual angle between two provided preferred directions. The parametrisation by local convected triads provides the maximum possible insight into the local mechanics relying on a phenomenologic theory. See also the full feathered examples in [31].

${ }^{5}$ With this assumption $\mathbf{g}$ satisfies the material impenetrability postulate, see (2.8). 
The last identity in (2.5) follows from the definition of duality and gives rise to the introduction of the symmetric and positive definite right Cauchy-Green tensor $\mathbf{C} \in$ $\operatorname{Sym}^{+}\left(T_{X}^{*} \mathscr{P}_{0} \otimes T_{X}^{*} \mathscr{P}_{0}\right)$;

$$
\mathbf{C}:=\mathbf{F}^{*} \mathbf{g F}: T_{X} \mathscr{P}_{0} \rightarrow T_{X}^{*} \mathscr{P}_{0},\left.\quad \mathbf{C}(\boldsymbol{\varphi})\right|_{\varphi=X}=\mathbf{G}(\boldsymbol{X}) .
$$

Finally, it is noted that the right Cauchy-Green tensor is the pull-back of the convected metric tensor $\mathbf{g}(\varphi)$ to the reference configuration. Moreover, the possibility of using it as a Lagrangian metric at $X \in T_{X} \mathscr{P}_{0}$ is noted. The postulate of material impenetrability takes the following form in convected frames,

$$
J(\varphi):=\sqrt{\operatorname{det}\left[\mathbf{G}^{-1} \mathbf{C}(\varphi)\right]}=\sqrt{G^{-1} g(\varphi)}>0,
$$

where $J$ is the volume ratio. Further, the stretch $\lambda_{\mathrm{F}}$ of a material line element in the material direction $\boldsymbol{G}_{\mathrm{F}} \in T_{\boldsymbol{X}} \mathscr{P}_{0}$ is expressed in terms of $\mathbf{C}$ as $\lambda_{\mathrm{F}}(\mathbf{C})=\sqrt{\left\|\boldsymbol{G}_{\mathrm{F}}\right\|_{\mathbf{C}}^{2}}$, where the right handside is an example of using $\mathbf{C}$ as the Lagrangian metric, cf. (2.5). Following Spencer [16], Boehler [17] and Zhang and Rychlewski [18], a stress-like Lagrangian structural tensor,

$$
\mathbf{A}:=\hat{\boldsymbol{M}} \otimes \hat{\boldsymbol{M}} \in \operatorname{Sym}\left(T_{X} \mathscr{P}_{0} \otimes T_{X} \mathscr{P}_{0}\right),
$$

is determined by a given preferred direction $\hat{\boldsymbol{M}}$. Here, it will be employed as a basis vector in the local triad, i.e., $\hat{\boldsymbol{M}} \in\left\{\boldsymbol{G}_{A}\right\}$, by construction. A structural tensor is idempotent, $\mathbf{A}_{\mathrm{F}}^{2}=\mathbf{A}_{\mathrm{F}}$, which follows from the normalisation, $\left\|\boldsymbol{G}_{\mathrm{F}}\right\|=1$.

Further, the standard set of admissible transplacements $\varphi(X, t)$ is defined by,

$$
\mathscr{C}:=\left\{\varphi(X, t): \mathscr{P}_{0} \rightarrow \mathscr{P} \mid J(\varphi)>0 \text { in } \mathscr{P}_{0}, \quad \text { and } \quad \boldsymbol{\varphi}=\bar{\varphi} \quad \text { on } \partial \mathscr{P}_{0 \mathrm{D}}\right\},
$$

where $\partial \mathscr{P}_{0 \mathrm{D}}$ denotes the part of the boundary where the transplacement is prescribed.

Moreover, the tangent set to $\mathscr{C}$ is introduced as the standard set of admissible material velocities, $\dot{\varphi}(\boldsymbol{X}, t)$;

$$
T_{\varphi} \mathscr{C}:=\left\{\dot{\boldsymbol{\varphi}}(\boldsymbol{X}, t): \mathscr{P}_{0} \rightarrow \mathscr{P} \mid\left\langle\dot{\mathbf{C}}, J^{2} \mathbf{C}^{-1}\right\rangle>0 \text { in } \mathscr{P}_{0}, \quad \text { and } \quad \dot{\boldsymbol{\varphi}}=\mathbf{0} \quad \text { on } \quad \partial \mathscr{P}_{0 \mathrm{D}}\right\} .
$$

It is also known as the standard set of admissible variations due to the connections $\delta \varphi \cong \Delta \varphi=\dot{\varphi} \Delta t$, see Simo and Hughes [24, Sect. 7.2.1]. Thus, a material velocity (increment or variation) must respect homogeneous Dirichlet boundary conditions in order to be identified with a test function or variation in the standard Bubnov-Galerkin sense. This requirement becomes clear linearising a weak formulation. ${ }^{6}$ The rate form (or first variation) of the condition of impenetrability $\dot{J}(\varphi)>0$, which here is expressed in terms of the strain rate $\dot{\mathbf{C}}=D \mathbf{C}(\varphi)[\dot{\varphi}]$ at $\varphi \in \mathscr{C}$. Finally, we may note that $\mathscr{C}$ is a submanifold of the Euclidean point manifold $\mathscr{E}$ and that $T_{\varphi} \mathscr{C}$ is a submanifold of the associated translation space $\mathscr{U}$.

\section{Simple Kinematic Internal Constraints}

In this section the kinematic aspects of subsidiary kinematic conditions are considered. Consequences on the work conjugate stresses are derived from the constraint rates.

\footnotetext{
${ }^{6}$ A prescribed transplacement $\bar{\varphi} \in \partial \mathscr{P}_{0 \mathrm{D}}$ is imposed computing its corresponding reaction. Thus, $\bar{\varphi}(X, t) \notin$ $T_{\varphi} \mathscr{C}$ is a given vector field which may vary with time.
} 


\subsection{Constraint Manifold Theory}

This subsection is essentially an adaption of the constraint manifold theory following PodioGuidugli and associates [4-6]. Some observations are added. The constraint manifold is embedded into the set of admissible transplacements (2.10) and its tangent is embedded into the set of admissible variations (2.11). It provides a bridge to computational mechanics. The embedding couples the restrictions imposed by a kinematic constraint on the strain $\mathbf{C}(\varphi)$ and its rate or first variation, to the transplacement $\varphi$ and its rate $\dot{\varphi}$ or variation $\delta \varphi$, respectively.

Many materials vigorously resist certain transplacements to such an extent that it a perfect approximation to exclude them from the standard set of admissible transplacements $(2.10) \mathscr{C}(\boldsymbol{\varphi})$. Classic examples concern, pure dilatation $J^{1 / 3}(\mathbf{C})$ and uniaxial stretch $\lambda_{\mathrm{F}}\left(\mathbf{C} ; \mathbf{A}_{\mathrm{F}}\right)$ in a material direction given by $\mathbf{A}_{\mathrm{F}}$. An exclusion of a class of transplacements from $\mathscr{C}$ may provide considerable simplification in solving boundary-value problems analytically, while the same restriction may call for a special formulation solving them numerically. Incompressibility is the leading example with vast literature in both branches. The kind of additional subsidiary kinematic conditions considered here, appended to the set of admissible transplacements $\mathscr{C}$, are called internal constraints, since they act in the domain occupied by the body, and in addition require a modification of the material description. Here we consider the restriction of the purely mechanical theory of a non-aging, i.e., time invariant, so-called simple material in the sense of Noll $[19,20]$, to the subclass of hyperelasticity. Basically, for hyperelasticity, there are three material stretches and three direction cosines in the actual placement that may be constrained point-wise, see the convected metric tensor $\mathbf{g}(\boldsymbol{\varphi})$ (2.6). That is, we need to consider the actual value of its Eulerian form $\mathbf{g}(\boldsymbol{\varphi})$, or equivalently, its Lagrangian form in the reference placement, $\mathbf{C}(\varphi)$, at $\varphi(X, \tau)$ for $\tau=t$. In order to determine the work-less and the work performing stresses the strain rate $\dot{\mathbf{C}}$ is needed. Simple internal constraints in conjunction with thermoelasticity and in general thermodynamics for simple materials are out of the scope for this contribution. Extensions are dealt with by, Gurtin and Podio-Guidugli [5], Negahban [10] and by Bertram and Glüge [8], respectively.

The dimension of symmetric tensors is six. Consequently, there can be at most six independent internal constraints simultaneously. A fully constrained material is rigid. The convected metric tensor for a rigid material coincides with the referential metric tensor $\mathbf{G}$ for $t \geq 0$. An isotropic internal constraint can be expressed in terms of the principal invariants of the Lagrangian metric tensor $\mathbf{C}$ alone, while an anisotropic internal constraint, in the sense of Spencer [16], involves the joint invariants of $\mathbf{C}$ and a set of structural tensors defining the anisotropy, of the type (2.9).

Definition 3.1 (Simple internal constraint as a constraint manifold) Following PodioGuidugli and associates [4-6], a simple, time invariant, internal constraint, may be considered in terms of a differentiable manifold,

$$
\mathscr{M}_{\phi}\left(\boldsymbol{\varphi}, \phi^{0}, \mathscr{S}\right):=\left\{\mathbf{C}(\boldsymbol{\varphi}) \in \operatorname{Sym}^{+}\left(T_{X}^{*} \mathscr{P}_{0} \otimes T_{X}^{*} \mathscr{P}_{0}\right) \mid \phi(\mathbf{C} ; \mathscr{S})=\phi^{0}, \quad \boldsymbol{\varphi} \in \mathscr{C}\right\},
$$

where $\varphi \in \mathscr{C}$ is an admissible transplacement, and where the constrained quantity, $\phi$, takes on the given constant value $\phi^{0}:=\phi(\mathbf{G}(\boldsymbol{X}) ; \mathscr{S}) \in \mathbb{R}$ it has in the referential placement, see (2.7). Further, $\mathscr{S}$ is a non-empty set, containing the given referential metric tensor $\mathbf{G}^{-1}$ and possibly a given referential structural tensor field, e.g., in the form (2.9). See [16-18] for the use of structural tensors for anisotropic solids. Finally, the function $\phi$ is supposed to be continuously differentiable sufficiently many times with respect to $\mathbf{C}$. 
The following basic two families of constrained quantities $\phi_{i}(\mathbf{C}(\varphi)) \in \mathscr{F}_{i}, i=1$ or $i=2$ are used:

$$
\mathscr{F}_{1}:=\left\{\lambda_{1}, \lambda_{2}, \lambda_{3}, \alpha_{12}, \alpha_{13}, \alpha_{23}\right\} \quad \text { or } \quad \mathscr{F}_{2}:=\left\{J, \bar{\lambda}_{1}, \bar{\lambda}_{2}, \bar{\lambda}_{3}, \alpha_{12}, \alpha_{13}, \alpha_{23}\right\}
$$

where $\bar{\lambda}_{a}:=J^{-1 / 3} \lambda_{a}, a=1,2,3$ are the so-called volume-preserving stretches. These two families separate the classes of compressible and incompressible materials, see [29]. The second family is subject to the normalisation condition $\operatorname{det}\left[J^{-1 / 3} \mathbf{F}\right] \equiv 1$. A collection of $1<n \leq 6$ compatible simple internal constraints is denoted the constraint manifold,

$$
\mathscr{M}:=\bigcap_{i=1}^{n} \mathscr{M}_{\phi_{i}}\left(\boldsymbol{\varphi}, \phi^{0}, \mathscr{S}_{i}\right),
$$

see also Steigmann [11]. In passing it is noted that $\mathbf{C}$ is frame invariant and that the presence of the referential metric tensor $\mathbf{G}^{-1}$ implies that the identity mapping $\mathbf{I} \in \mathscr{M}$.

Finally, a simple minded view suggests to consider a kinematic constraint $\phi(\mathbf{C} ; \mathscr{S})=$ $\phi^{0}$ as a level set in $\mathbb{R}^{6}$. The recent contribution by Vianello [6] discourages from such a departure, however.

Just as a function $F: \mathscr{E} \rightarrow \mathbb{R}$ of the form $F(\boldsymbol{X})=0$ embedded in the standard Euclidean point space $\mathscr{E}$ defines a surface $\mathscr{Y}:=\{\boldsymbol{X} \in \mathscr{E} \mid F(\boldsymbol{X})=0\}$, i.e., a set of points in three space, $\mathbb{R}^{3}$. Like-wise, equation (3.1) defines a hyper-surface, $\mathscr{M}_{\phi}: \mathbf{C}(\varphi) \in \operatorname{Sym}^{+}\left(T_{X}^{*} \mathscr{P}_{0} \otimes\right.$ $\left.T_{X}^{*} \mathscr{P}_{0}\right) \rightarrow \mathbb{R}$ of admissible strains in $\mathbb{R}^{6}$, suggesting restrictions of $\mathscr{C},(2.10)$. From a simple minded geometric point of view, a constraint may be seen as an iso-surface in $\mathbb{R}^{6}$. There are however cases where this analogy breaks down, see [6].

Definition 3.2 (Augmented set of admissible transplacements) A constraint manifold (3.3) $\mathscr{M}$, is here added to the standard set of admissible transplacements $(2.10), \mathscr{C}$, forming the augmented set of admissible transplacements,

$$
\mathscr{C}_{\mathrm{a}}:=\{\varphi(X, t) \in \mathscr{C} \mid \mathbf{C}(\varphi) \in \mathscr{M}\} .
$$

The requirements of linear independence of multiple constraints from Carlson et al. [7] and Negahban [10] are recalled. Reinforcement with inextensible fibre families, requires that their preferred directions are distinct.

Definition 3.3 (Linear independent constraints) A number of constraints $\phi_{i}(\mathbf{C})=\phi_{i}^{0}$ are independent if and only if their gradients $\partial_{\mathbf{C}} \phi_{i}, i=1,2, \ldots, n \leq 6$ define a linear independent set of tensors. That is, if their linear combination is the zero tensor, if and only if all scalar multipliers are zero;

$$
\sum_{i=1}^{n} \alpha_{i} \partial_{\mathbf{C}} \phi_{i}=\mathbf{0} \quad \Leftrightarrow \quad \forall \alpha_{i}=0 .
$$

In analogy with the gradient $\partial_{\boldsymbol{X}} F(\boldsymbol{X})$ to a surface $F(\boldsymbol{X})=0$ at $\boldsymbol{X}$ in $\mathbb{R}^{3}$, the tensor gradient $\partial_{\mathbf{C}} \phi(\mathbf{C})$ to the constraint $\phi(\mathbf{C})=\phi^{0}$ at $\mathbf{C}$ in $\mathbb{R}^{6}$, may be conceived as a normal, respectively. 
Definition 3.4 (The set of normals, $N_{\mathbf{C}} \mathscr{M}$ ) For an independent set of tensor gradients,

$$
\partial_{\mathbf{C}} \phi_{i}(\mathbf{C}) \in \operatorname{Sym}\left(T_{X} \mathscr{P}_{0} \otimes T_{\boldsymbol{X}} \mathscr{P}_{0}\right), \quad i=1,2, \ldots, n \leq 6,
$$

the set of normals to the constraint manifold $\mathscr{M}$ evaluated for a $\varphi \in \mathscr{C}_{\mathrm{a}}$ at $\mathbf{C}(\boldsymbol{\varphi}),(3.4)$, is recalled, cf. [7, Eqs. (2.4)]:

$$
N_{\mathbf{C}} \mathscr{M}:=\operatorname{Span}\left\{\partial_{\mathbf{C}} \phi_{i}(\mathbf{C}(\varphi)) \in \operatorname{Sym}\left(T_{\boldsymbol{X}} \mathscr{P}_{0} \otimes T_{\boldsymbol{X}} \mathscr{P}_{0}\right) i=1,2, \ldots, n \leq 6, \quad \boldsymbol{\varphi} \in \mathscr{C}_{\mathrm{a}}\right\} .
$$

Note that for a simple inextensibility in the preferred direction $\hat{\boldsymbol{M}}(\boldsymbol{X})$, the normal to the inextensibility constraint is $\mathbf{A}(\boldsymbol{X})$ see (2.9).

Further, the time rate of a constraint is essential for the development. It is computed by the chain rule of differential calculus as,

$$
\dot{\phi}=\left\langle D \mathbf{C}(\boldsymbol{\varphi})[\dot{\boldsymbol{\varphi}}], \frac{\partial \phi(\mathbf{C})}{\partial \mathbf{C}}\right\rangle=0 \quad \text { at } \boldsymbol{\varphi} \in \mathscr{C}_{\mathrm{a}} .
$$

Here $D \mathbf{C}(\varphi)[\dot{\varphi}]$ is the first variation of the right Cauchy-Green stretch tensor at $\varphi$ in the direction of the material velocity $\dot{\varphi}$. It provides a constraint on $\dot{\varphi}(X, t)$ or equivalently on the strain rate at $\varphi, \dot{\mathbf{C}}(\varphi)=D \mathbf{C}(\varphi)[\dot{\varphi}]$. The collection of the constraints on the strain rate is added to the standard set of admissible material velocities (2.11).

Definition 3.5 (The tangent sets $T_{\mathbf{C}} \mathscr{M}$ and $T_{\varphi} \mathscr{C}_{\mathrm{a}}$ ) First, the tangent set, corresponding to the normal set $N_{\mathbf{C}} \mathscr{M}$, is recalled, cf. [7, Eqs. (2.4) and (2.5)],

$$
T_{\mathbf{C}} \mathscr{M}:=\left\{\dot{\mathbf{C}} \in \operatorname{Sym}\left(T_{X}^{*} \mathscr{P}_{0} \otimes T_{\boldsymbol{X}}^{*} \mathscr{P}_{0}\right) \mid\left\langle\dot{\mathbf{C}}, \partial_{\mathbf{C}} \phi_{i}\right\rangle=0, \forall \partial_{\mathbf{C}} \phi_{i} \in N_{\mathbf{C}} \mathscr{M}, i=1,2, \ldots, n \leq 6,\right\},
$$

where the strain rate $\dot{\mathbf{C}}(\varphi)=D \mathbf{C}(\varphi)[\dot{\varphi}]$ is computed at $\varphi \in \mathscr{C}_{\mathrm{a}}$. Then, the augmented set of admissible material velocities which may be called the augmented set of admissible variations is formed as,

$$
T_{\varphi} \mathscr{C}_{\mathrm{a}}:=\left\{\dot{\boldsymbol{\varphi}}(\boldsymbol{X}, t) \in T_{\varphi} \mathscr{C} \mid D \mathbf{C}(\boldsymbol{\varphi})[\dot{\boldsymbol{\varphi}}] \in T_{\mathbf{C}} \mathscr{M}\right\} .
$$

The definition of a stress tensor which is workless in all admissible transplacements $\boldsymbol{\varphi}(\boldsymbol{X}, t) \in \mathscr{C}_{\mathrm{a}}$ with admissible material velocity $\dot{\boldsymbol{\varphi}}(\boldsymbol{X}, t) \in T_{\varphi} \mathscr{C}_{\mathrm{a}}$ at $\boldsymbol{X} \in \mathscr{P}_{0}$ corresponding locally to a stretch rate $D \mathbf{C}(\varphi)[\dot{\varphi}] \in T_{\mathbf{C}} \mathscr{M}$ is also needed. The phenomenologic description of kinematics in the presence of simple internal constraints is completed. The workless stress, in other words the stress reaction, is neither an element of the kinematics nor of the constitutive part of the stress response. It is energy conjugate to the kinematically inadmissible modes of deformation, akin to reactions in rigid-body mechanics.

Definition 3.6 (Workless stress tensor) For any admissible material velocity $\dot{\boldsymbol{\varphi}}(\boldsymbol{X}, t) \in T_{\varphi} \mathscr{C}_{\mathrm{a}}$ corresponding to an admissible strain rate $\dot{\mathbf{C}} \in T_{\mathbf{C}} \mathscr{M}$ (Definition 3.5), and a tensor $\mathbf{S}_{\mathrm{R}} \in N_{\mathbf{C}} \mathscr{M}$ (Definition 3.4), the vanishing of the scalar product,

$$
0=\left\langle\dot{\mathbf{C}}, \mathbf{S}_{\mathrm{R}}\right\rangle: T_{\mathbf{C}} \mathscr{M} \times N_{\mathbf{C}} \mathscr{M} \rightarrow \mathbb{R},
$$

defines an energy conjugate workless stress tensor which, for a set of (Lagrange) multipliers $\varrho^{i} \in \mathbb{R}, i=1,2, \ldots, n \leq 6$, has the representation,

$$
\mathbf{S}_{\mathrm{R}}=\sum_{i=1}^{n} 2 \varrho^{i} \partial_{\mathbf{C}} \phi_{i}
$$


For $n=6$ any material is rigid. There is no stretch or shear $\mathbf{C}=\mathbf{G}$. The stress response is in the form (3.10). For $n<6$, the difference between the total stress ${ }^{7}$ and the workless stress, $\mathbf{S}-\mathbf{S}_{\mathrm{R}}=: \mathbf{S}_{\mathrm{E}}$ may be non-trivial, for non-trivial $\mathbf{C} \in \mathscr{M}$. According to the principle of determinism for simple materials subject to internal constraints [1, Sect. 30, p. 70] the so-called extra stress $\mathbf{S}_{\mathrm{E}}$ can be computed from the history of the right Cauchy-Green stretch tensor $\mathbf{C}^{t}(s) \in \mathscr{M}$ for objective simple materials in the sense of Noll [20], see also Day [19]. For hyperelasticity we only need that $\mathbf{C}(t) \in \mathscr{M}$ at the current instant $t$ and that $\dot{\mathbf{C}}(t) \in T_{\mathbf{C}} \mathscr{M}$. The proposed additive decomposition of the total stress [1, 3, 7] follows from the projection theorem in linear algebra Bowen and Wang [21, Th. 17.3] (provided in Appendix D) applied to the set of symmetric tensors $\mathbf{S} \in \operatorname{Sym}\left(T_{X} \mathscr{P}_{0} \otimes T_{X} \mathscr{P}_{0}\right)$, as the direct sum $\operatorname{Sym}=\operatorname{Sym}_{\mathrm{R}} \oplus \mathrm{Sym}_{\mathrm{E}}$. The classic theory [1] is coherent with the constraint manifold theory $[3,7]$. The latter goes one step further. It removes a possible scalar valued indeterminacy in the extra stress $\mathbf{S}_{\mathrm{E}}{ }^{8}$ by normalisation. The so-called active stress $\mathbf{S}_{\mathrm{A}}:=\mathbf{S}-\mathbf{S}_{\mathrm{R}}$ is introduced. There, by postulate, $\mathbf{S}_{\mathrm{A}}$ is constructed orthogonal to $\mathbf{S}_{\mathrm{R}}$ in the sense of the Lagrangian inner product $\left(\mathbf{S}_{\mathrm{R}}, \mathbf{S}_{\mathrm{A}}\right)_{\left(T_{\boldsymbol{X}} \mathscr{P}_{0}, \mathbf{C}\right)}=0$, see Appendix B. Imposing this scalar valued subsidiary condition makes the stress decomposition unique.

\subsection{Construction of a Constrained Cauchy-Green/Lagrangian-Metric Tensor}

In this subsection the procedure to construct a constrained Cauchy-Green tensor denoted $\hat{\mathbf{C}} \in \mathscr{M}$ by modifying a given actual Cauchy-Green tensor $\mathbf{C}$ is presented, i.e., item $\mathscr{C}_{1}$ in the Introduction, the first of the main contributions of this work. The resulting constructed tensor $\hat{\mathbf{C}}=\hat{\mathbf{C}}(\mathbf{C})$ induces a unique direct sum decomposition of the total stress into workless and work performing parts of $\mathbf{S}_{\mathrm{R}}$ and $\mathbf{S}_{\mathrm{A}}$ corresponding to the actual constraint manifold. Using the constrained Cauchy-tensor bypasses the need to assume a normalisation condition, e.g. $\left(\mathbf{S}_{\mathrm{R}}, \mathbf{S}_{\mathrm{A}}\right)_{\mathrm{C}}=0$ as in $[3,7]$. In view of the definition $(2.7)$, the constrained Cauchy-Green tensor $\hat{\mathbf{C}}$ may be interpreted as a constrained convected Lagrangian metric tensor. The constrained convected Eulerian metric tensor denoted $\hat{\mathbf{g}}$ is obtained simultaneously.

Definition 3.7 (Constrained Cauchy-Green tensor) For a given arbitrary Cauchy-Green tensor $\mathbf{C}$, a constrained Cauchy-Green tensor $\hat{\mathbf{C}}=\hat{\mathbf{C}}(\mathbf{C})$ is constructed such that,

$$
\hat{\mathbf{C}}(\mathbf{C})=\hat{\mathbf{C}} \in \mathscr{M}, \quad \forall \mathbf{C} \in \operatorname{Sym}\left(T_{X}^{*} \mathscr{P}_{0} \otimes T_{X}^{*} \mathscr{P}_{0}\right) .
$$

Remark: In view of the Definition (3.1) the constructed Cauchy-Green tensor satisfies each constraint as an identity; i.e., $\phi_{i}(\hat{\mathbf{C}}(\mathbf{C}) ; \mathscr{S}) \equiv \phi_{i}^{0}$ for $i=1,2, \ldots, n$. Further, $\hat{\mathbf{C}}$ has the following two properties,

$$
\left.\begin{array}{rl}
\hat{\mathbf{C}}(\mathbf{C}) & =\mathbf{C}, \quad \text { for given } \quad \mathbf{C} \in \mathscr{M} \\
\frac{\partial \hat{\mathbf{C}}}{\partial \phi_{i}} & =\mathbf{0}, \quad i=1,2, \ldots, n
\end{array}\right\}
$$

(3.12) $)_{1}$ implies that no modification is performed if given $\mathbf{C} \in \mathscr{M}$, and where (3.12) 2 implies that the constrained convected metric $\hat{\mathbf{C}} \in \mathscr{M}$ does not depend on any of the constrained quantities.

\footnotetext{
${ }^{7}$ In the sense of the second Cauchy stress principle [1, Eq. (16.5)].

${ }^{8}$ E.g. the trace of $\mathbf{S}_{\mathrm{E}}$ in incompressibility.
} 
Let $\mathbf{f}_{i}\left(\phi_{i}\right): T_{\varphi(X)} \mathscr{P} \rightarrow T_{\varphi(X)} \mathscr{P}$ be a bijective linear transformation on the actual placement depending on the single constrained quantity $\phi_{i} \in \mathscr{F}_{1}$ or $\phi_{i} \in \mathscr{F}_{2}$, of the type defined by Definition 3.1. The composition of $n \leq 6$ such mappings is introduced:

$$
\mathbf{f}:=\prod_{i=1}^{n} \mathbf{f}_{i}\left(\phi_{i}\right) \in \operatorname{Lin}^{+}\left(T_{\varphi(X)} \mathscr{P} \otimes T_{\varphi(X)}^{*} \mathscr{P}\right) .
$$

Proposition 3.1 (Construction of a constrained Lagrangian convected metric) Given the constraint manifold in the sense of Vianello [6] $\mathscr{M}$, the mapping $\mathbf{f}(3.13)$, the actual tangent map $\mathbf{F}$ and the background convected metric tensor $\mathbf{g}(\boldsymbol{\varphi})$; the following composition defines the admissible tangent map:

$$
\mathbf{f}^{-1} \mathbf{F}=: \hat{\mathbf{F}} \quad \text { such that } \hat{\mathbf{F}}^{*} \mathbf{g}(\boldsymbol{\varphi}) \hat{\mathbf{F}} \in \mathscr{M}
$$

where the associated constrained Eulerian and Lagrangian convected metric tensors are identified;

$$
\hat{\mathbf{C}}:=\mathbf{F}^{*} \hat{\mathbf{g}}(\varphi) \mathbf{F} \quad \text { where } \hat{\mathbf{g}}(\varphi):=\mathbf{f}^{-*} \mathbf{g}(\varphi) \mathbf{f}^{-1}
$$

Proof In Appendix $\mathrm{C}$ it is shown by calculation that $\hat{\mathbf{C}} \in \mathscr{M}$ for the three basic cases incompressibility, inextensibility and preservation of angle.

The constrained Lagrangian convected metric/Cauchy-Green tensor $\hat{\mathbf{C}} \in \mathscr{M}$ provides the proper referential objective strain measure for the internally constrained situation.

Example 1 (Incompressibility) See Appendix C.1. The normalisation by $J^{-2 / 3}>0, \overline{\mathbf{C}}:=$ $\mathbf{F}^{*} J^{-2 / 3} \mathbf{g F}$, evidently introduces the volume preserving stretches $\bar{\lambda}_{a}:=J^{-1 / 3} \lambda_{a}, a=1,2,3$, producing the isochoric spatial metric $\overline{\mathbf{g}}:=J^{-2 / 3} \mathbf{g}$, confer (2.6). The constrained Lagrangian metric tensor is the unimodular right Cauchy-Green stretch tensor, $\hat{\mathbf{C}}=\left[\phi^{0}\right]^{2 / 3} \overline{\mathbf{C}}=\overline{\mathbf{C}}$, where the referential value $\phi^{0}=\operatorname{det}\left[\mathbf{G}^{-1} \mathbf{C}\right]_{\mathbf{C}=\mathbf{G}}=1$.

Example 2 (Two oblique plain extensibility constraints) See Appendix C.2. For two oblique plain extensibility constraints, $\phi_{i}\left(\mathbf{C} ; \mathbf{G}^{-1}, \mathbf{A}_{\mathrm{i}}\right)=\phi_{i}^{0}, \phi_{i}=\lambda_{\mathrm{i}}$ and $\phi_{i}^{0}=1$ for $i=1,2$, the constrained Lagrangian metric tensor becomes;

$$
\begin{aligned}
\hat{\mathbf{C}}:=\mathbf{F}^{*} \hat{\mathbf{g}} \mathbf{F}= & \mathbf{C}+\sum_{\mathrm{F}=1}^{2}\left(\left[\phi_{\mathrm{F}}^{0}\right]^{2}-\lambda_{\mathrm{F}}^{2}\right)\left(\underline{G}^{\mathrm{F}} \otimes \underline{\sim}^{\mathrm{F}}\right) \\
& +\alpha_{12}\left(\phi_{1}^{0} \phi_{2}^{0}-\lambda_{1} \lambda_{2}\right) 2 \operatorname{sym}\left\{{\underset{\sim}{\boldsymbol{G}}}^{1} \otimes \underline{\sim}^{2}\right\} \\
& +\alpha_{13}\left(\phi_{1}^{0}-\lambda_{1}\right) \lambda_{3} 2 \operatorname{sym}\left\{\underset{\sim}{\left.\boldsymbol{G}^{1} \otimes \underline{\sim}^{3}\right\}}\right. \\
& +\alpha_{23}\left(\phi_{2}^{0}-\lambda_{2}\right) \lambda_{3} 2 \operatorname{sym}\left\{\underset{\sim}{\left.\boldsymbol{G}^{2} \otimes \underset{\sim}{\boldsymbol{G}^{3}}\right\}}\right. \\
= & {\left[\begin{array}{ccc}
{\left[\phi_{1}^{0}\right]^{2}} & \alpha_{12} \phi_{1}^{0} \phi_{2}^{0} & \alpha_{13} \phi_{1}^{0} \lambda_{3} \\
\bullet & {\left[\phi_{2}^{0}\right]^{2}} & \alpha_{23} \phi_{2}^{0} \lambda_{3} \\
\bullet & \bullet & \lambda_{3}^{2}
\end{array}\right]_{a b} \delta_{A}^{a} \delta_{B}^{a} \boldsymbol{G}^{A} \otimes{\underset{\sim}{G}}^{B} . }
\end{aligned}
$$


Example 3 (Angle preservation/unshearability) See Appendix C.3. The constraint fixing the referential direction cosine $\phi^{0}=\alpha_{a b}^{0}$ between two material line elements $\boldsymbol{G}_{a}$ and $\boldsymbol{G}_{b}$ in $T_{X} \mathscr{P}_{0}$ is;

$$
\phi\left(\mathbf{C} ; \mathbf{G}^{-1}, \boldsymbol{G}_{a} \otimes \boldsymbol{G}_{b}\right):=\alpha_{a b}=\alpha_{a b}^{0} .
$$

Note that $\boldsymbol{G}_{a}$ and $\boldsymbol{G}_{b}$ are employed as referential basis vectors and that the second parametric argument is a structural tensor in the sense of Spencer [16]. The annihilation mapping for this case $\mathbf{f}\left(\gamma_{a b} ; \boldsymbol{g}_{a}, \underline{g}^{b}\right): T_{\varphi(\boldsymbol{X})} \mathscr{P} \rightarrow T_{\boldsymbol{\varphi}(\boldsymbol{X})} \mathscr{P}$ and its inverse is formed in terms of the spatial natural convected basis vectors: ${ }^{9}$

$$
\left.\begin{array}{l}
\mathbf{f}\left(\gamma_{a b} ; \boldsymbol{g}_{a}, \underset{\sim}{\boldsymbol{g}^{b}}\right):=\mathbf{1}+\gamma_{a b} \boldsymbol{g}_{a} \otimes \underset{\sim}{\boldsymbol{g}^{b}}, \quad \operatorname{det} \mathbf{f}=1, \\
\mathbf{f}^{-1}\left(\gamma_{a b} ; \underset{a}{\boldsymbol{g}_{a}}, \underset{\sim}{\boldsymbol{g}^{b}}\right):=\mathbf{1}-\gamma_{a b} \boldsymbol{g}_{a} \otimes \underset{\sim}{\boldsymbol{g}^{b}} .
\end{array}\right\}
$$

The expression for $\gamma_{a b}$ is;

$$
\gamma_{a b}=\left(\alpha_{a b}-\alpha_{a b}^{0}\right) \frac{\lambda_{b}}{\lambda_{a}} .
$$

For $a=1$ and $b=2$ the resulting constrained Lagrangian metric tensor is obtained as:

$$
\begin{aligned}
& \hat{\mathbf{C}}=\mathbf{F}^{*} \hat{\mathbf{g}} \mathbf{F}, \quad \hat{\mathbf{g}}=\mathbf{f}^{-*} \mathbf{g f}^{-1}, \\
& \hat{\mathbf{g}} \stackrel{(3.17)}{=}\left[\begin{array}{ccc}
\lambda_{1}^{2} & \alpha_{12}^{0} \lambda_{1} \lambda_{2} & \alpha_{13} \lambda_{1} \lambda_{3} \\
\bullet & \lambda_{2}^{2} & \alpha_{23} \lambda_{2} \lambda_{3} \\
\bullet & \bullet & \lambda_{3}^{2}
\end{array}\right] \underset{a b}{{\underset{\sim}{g}}^{a} \otimes \underset{\sim}{\mathbf{g}^{b}} .}
\end{aligned}
$$

Further, using the constrained convected metric approach the constraint rates are identically satisfied, by construction.

Proposition $3.2\left(\mathrm{~d}_{\mathrm{t}} \hat{\mathbf{C}} \in T_{\mathbf{C}} \mathscr{M}\right)$ The strain rate $\mathrm{d}_{\mathrm{t}} \hat{\mathbf{C}}$ computed from the constructed constrained convected metric tensor $\hat{\mathbf{C}} \in \mathscr{M}$ is an element in the tangent set, i.e.,

$$
\mathrm{d}_{\mathrm{t}} \hat{\mathbf{C}} \in T_{\mathbf{C}} \mathscr{M}
$$

Proof Time differentiating the defining identity of $\hat{\mathbf{C}}(3.11), \phi_{i}(\hat{\mathbf{C}}) \equiv \phi_{i}^{0}$, yields,

$$
\mathrm{d}_{\mathrm{t}} \phi_{i}(\hat{\mathbf{C}})=\left\langle\mathrm{d}_{\mathrm{t}} \hat{\mathbf{C}}, \partial_{\hat{\mathbf{C}}} \phi_{i}\right\rangle \equiv 0 .
$$

Since, $\hat{\mathbf{C}} \in \mathscr{M}$, we have that $\partial_{\hat{\mathbf{C}}} \phi_{i} \in N_{\mathbf{C}} \mathscr{M}$ and in view of (3.22) the strain rate $\mathrm{d}_{\mathrm{t}} \hat{\mathbf{C}}$ is identically perpendicular to the constraint normal $\partial_{\hat{\mathbf{C}}} \phi_{i}$. Thus, $\mathrm{d}_{\mathrm{t}} \hat{\mathbf{C}} \in T_{\mathbf{C}} \mathscr{M}$.

On account of, Proposition 3.2 expanding the left hand-side of (3.22) and using (3.11) we have,

$$
\mathrm{d}_{\mathrm{t}} \hat{\mathbf{C}}(\mathbf{C}(\boldsymbol{\varphi}))=\hat{\mathbb{P}}^{*} \dot{\mathbf{C}}(\varphi) \in T_{\mathbf{C}} \mathscr{M}, \quad \text { where } \hat{\mathbb{P}}^{*}:=\partial_{\mathbf{C}} \hat{\mathbf{C}},
$$

\footnotetext{
${ }^{9}$ Without normalisation.
} 
for any admissible strain rate $\dot{\mathbf{C}}(\boldsymbol{\varphi})=D \mathbf{C}(\boldsymbol{\varphi})[\dot{\boldsymbol{\varphi}}] \in \operatorname{Sym}\left(T_{X}^{*} \mathscr{P}_{0} \otimes T_{X}^{*} \mathscr{P}_{0}\right), \boldsymbol{\varphi} \in \mathscr{C}$ and $\dot{\boldsymbol{\varphi}} \in$ $T_{\varphi} \mathscr{C}$. Inserting $(3.23)_{1}$ in (3.22) by duality we obtain,

$$
\left\langle\hat{\mathbb{P}}^{*} \dot{\mathbf{C}}(\varphi), \partial_{\hat{\mathbf{C}}} \phi_{i}\right\rangle=\left\langle\dot{\mathbf{C}}(\varphi), \hat{\mathbb{P}} \partial_{\hat{\mathbf{C}}} \phi_{i}\right\rangle \equiv 0 \Rightarrow \hat{\mathbb{P}} \partial_{\hat{\mathbf{C}}} \phi_{i}=\mathbf{0},
$$

for any constraint normal $\partial_{\hat{\mathbf{C}}} \phi_{i} \in N_{\mathbf{C}} \mathscr{M} \subset \subset \operatorname{Sym}\left(T_{X} \mathscr{P}_{0} \otimes T_{X} \mathscr{P}_{0}\right), i=1,2, \ldots, n$ and any admissible strain rate $\dot{\mathbf{C}}(\varphi) \in \operatorname{Sym}\left(T_{X}^{*} \mathscr{P}_{0} \otimes T_{X}^{*} \mathscr{P}_{0}\right)$. The implication of (3.24) is that the kernel of the fourth order tensor operator $\hat{\mathbb{P}}$ can be identified with the normal set, $\operatorname{Ker}(\hat{\mathbb{P}})=$ $N_{\mathbf{C}} \mathscr{M}$ since $\hat{\mathbf{C}} \in \mathscr{M}$. Further, time differentiating both sides of the identity (3.12) ${ }_{1}$ and using $(3.23)_{1}$ yields,

$$
\hat{\mathbb{P}}^{*} \dot{\mathbf{C}}=\dot{\mathbf{C}} \in T_{\mathbf{C}} \mathscr{M}
$$

Proposition 3.3 (Strain rate projection) $\hat{\mathbb{P}}^{*}$ is idempotent,

$$
\left[\hat{\mathbb{P}}^{*}\right]^{2}=\hat{\mathbb{P}}^{*}
$$

Proof Multiplying both sides of (3.25) by $\hat{\mathbb{P}}^{*}$ and simplifying proves the proposition for strain rates $\dot{\mathbf{C}} \in T_{\mathrm{C}} \mathscr{M}$ :

$$
\left(\left[\hat{\mathbb{P}}^{*}\right]^{2}-\hat{\mathbb{P}}^{*}\right) \dot{\mathbf{C}} \equiv 0 \Rightarrow\left[\hat{\mathbb{P}}^{*}\right]^{2}=\hat{\mathbb{P}}^{*}
$$

It follows that it is a projection on the space of strain rates:

$$
\hat{\mathbb{P}}^{*}: \operatorname{Sym}\left(T_{X}^{*} \mathscr{P}_{0} \otimes T_{X}^{*} \mathscr{P}_{0}\right) \rightarrow \operatorname{Sym}\left(T_{X}^{*} \mathscr{P}_{0} \otimes T_{X}^{*} \mathscr{P}_{0}\right)
$$

Further, it follows from the identity $\hat{\mathbb{P}}^{* *}=\hat{\mathbb{P}}$ that $\hat{\mathbb{P}}$ is also idempotent.

Proposition 3.4 (Stress projection) $\hat{\mathbb{P}}$ is a projection on the stress space:

$$
\hat{\mathbb{P}}: \operatorname{Sym}\left(T_{\boldsymbol{X}} \mathscr{P}_{0} \otimes T_{\boldsymbol{X}} \mathscr{P}_{0}\right) \rightarrow \operatorname{Sym}\left(T_{\boldsymbol{X}} \mathscr{P}_{0} \otimes T_{\boldsymbol{X}} \mathscr{P}_{0}\right) .
$$

Proof The result follows from inspection of the scalar product,

$$
\left\langle\dot{\mathbf{C}}, \hat{\mathbb{P}} \partial_{\mathbf{C}} \phi_{i}\right\rangle: T_{\mathbf{C}} \mathscr{M} \times N_{\mathbf{C}} \mathscr{M} \rightarrow \mathbb{R},
$$

where $\partial_{\mathbf{C}} \phi_{i} \in \operatorname{Sym}\left(T_{\boldsymbol{X}} \mathscr{P}_{0} \otimes T_{\boldsymbol{X}} \mathscr{P}_{0}\right)$ is stress-like.

Further, let subspaces $\operatorname{Sym}_{\mathrm{R}}$ and $\mathrm{Sym}_{\mathrm{A}}$ denote the kernel and the range of $\hat{\mathbb{P}}$ respectively. The projection theorem in linear algebra, provides the direct sum decomposition,

$$
\operatorname{Sym}=\operatorname{Sym}_{\mathrm{R}} \oplus \operatorname{Sym}_{\mathrm{A}} .
$$

Assuming the workless stress $\mathbf{S}_{\mathrm{R}}$ in the form (3.10) the constrained convected metric approach yields;

$$
\hat{\mathbb{P}} \mathbf{S}_{\mathrm{R}}=\mathbf{0},
$$

for a set of non-trivial scalars $\varrho^{i}, i=1,2, \ldots, n$.

One of the main results of this note can be stated. Namely, the constrained convected metric formulation induces a direct sum based decomposition of the stress. The result is stated by the following proposition; 
Proposition 3.5 (Stress decomposition) For any total stress tensor $\mathbf{S} \in \operatorname{Sym}\left(T_{X} \mathscr{P}_{0} \otimes\right.$ $\left.T_{\boldsymbol{X}} \mathscr{P}_{0}\right)$ and projection $\hat{\mathbb{P}}:=\left[\partial_{\mathbf{C}} \hat{\mathbf{C}}\right]^{*}$ by the projection theorem $[21$, Th. 17.3$]$ in linear algebra we have,

$$
\mathbf{S}=\mathbf{S}_{\mathrm{R}}+\mathbf{S}_{\mathrm{A}}
$$

Proof $\mathbf{S}_{\mathrm{A}}=\hat{\mathbb{P}} \mathbf{S} \in \operatorname{Sym}_{\mathrm{A}}$ and $\mathbf{S}_{\mathrm{R}}=(\mathbb{I}-\hat{\mathbb{P}}) \mathbf{S} \in$ Sym $_{\mathrm{R}}$ respectively.

Here, the workless stress $\mathbf{S}_{\mathrm{R}}$ is spanned by the normals to the constraint manifold using (3.10).

Note, that we have not yet involved any constitutive assumption for the complementary (working) stress $\mathbf{S}_{\mathrm{A}}$.

Next, the projection is constructed explicitly. To that end, both sides of the definition $\hat{\mathbb{P}}^{*}:=\partial_{\mathbf{C}} \hat{\mathbf{C}}$ are multiplied from the right by the tensor $\partial_{\phi_{i}} \mathbf{C}$ representing the sensitivity of the Lagrangian metric tensor with respect to the $i$-th constrained mechanical quantity. Simplifying using (3.12) 2 we obtain,

$$
\hat{\mathbb{P}}^{*} \partial_{\phi_{i}} \mathbf{C}=\mathbf{0}, \quad i=1,2, \ldots, n,
$$

which means that $\partial_{\phi_{i}} \mathbf{C}$ is in the left null-space of $\hat{\mathbb{P}}$.

An essential auxiliary result is derived.

Proposition 3.6 (Induced duality) The tensors $\partial_{\phi_{i}} \mathbf{C} \in T_{\mathbf{C}} \mathscr{M}$ and $\partial_{\mathbf{C}} \phi_{i} \in N_{\mathbf{C}} \mathscr{M}$ are dual:

$$
\left\langle\partial_{\phi_{i}} \mathbf{C}, \partial_{\mathbf{C}} \phi_{j}\right\rangle=\delta_{j}^{i}, \quad i, j=1,2, \ldots, n .
$$

Proof The result follows from evaluating the partial derivative, $\partial_{\phi_{i}} \phi_{j}$.

Its rationale is evident recalling that the constraints in $\mathscr{M}$ are required to be linearly independent.

The explicit form of the constrained convected metric induced stress projection $\hat{\mathbb{P}}$ can now be stated.

Proposition 3.7 (Explicit projection representation) The explicit form of the stress projection $\hat{\mathbb{P}}$ is;

$$
\hat{\mathbb{P}}:=\left[\frac{\partial \hat{\mathbf{C}}}{\partial \mathbf{C}}\right]^{*} \equiv \mathbb{I}-\sum_{i=1}^{n} \frac{\partial \phi_{i}}{\partial \mathbf{C}} \otimes \frac{\partial \mathbf{C}}{\partial \phi_{i}} .
$$

Proof It is deduced using the definition of the null-space (3.24) and that of the left nullspace implied by (3.31). Using (3.32) it is readily checked that it is idempotent and has the right kernel and range.

Example 1 (Incompressibility) For incompressibility; $\phi=J(\mathbf{C}), \mathbf{C}=J^{2 / 3} \overline{\mathbf{C}}$ which yields $\partial_{J} \mathbf{C}={ }^{2 / 3} J^{-1} \mathbf{C}$ and $\partial_{\mathbf{C}} J=1 / 2 J \mathbf{C}^{-1}$. Inserting in (3.33) gives,

$$
\hat{\mathbb{P}}=\mathbb{I}-\frac{1}{3} \mathbf{C}^{-1} \otimes \mathbf{C},
$$

which is recognized as the deviatoric projection, and which renders the working stress $\mathbf{S}_{\mathrm{A}}$ traceless. 
Example 2 (Two oblique plain extensibility constraints) For two oblique plain extensibility constraints the explicit expression for the stress projection $\hat{\mathbb{P}}$ becomes,

$$
\hat{\mathbb{P}}=\mathbb{I}-\sum_{\mathrm{F}=1}^{2} \frac{\partial \lambda_{\mathrm{F}}}{\partial \mathbf{C}} \otimes \frac{\partial \mathbf{C}}{\partial \lambda_{\mathrm{F}}},
$$

where

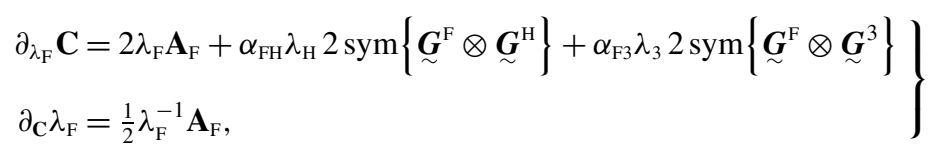

where $H=2,1$ for $F=1,2$. Using idempotency and the left null-space property (3.31) the working stress $\mathbf{S}_{\mathrm{A}}=\hat{\mathbb{P}} \mathbf{S}$ becomes complementary to the workless stress (Definition 3.6), see Proposition 3.5 .

Example 3 (Angle preservation/unshearability) For the angle preservation constraint (3.17) with $a=1$ and $b=2$ the stress projection $\hat{\mathbb{P}}$ assumes the form,

$$
\hat{\mathbb{P}}=\mathbb{I}-\frac{\partial \alpha_{12}}{\partial \mathbf{C}} \otimes \frac{\partial \mathbf{C}}{\partial \alpha_{12}},
$$

where

$$
\begin{aligned}
& \frac{\partial \alpha_{12}}{\partial \mathbf{C}}=\frac{1}{2}\left[\lambda_{1}^{-1} \lambda_{2}^{-1}\left(\boldsymbol{G}_{1} \otimes \boldsymbol{G}_{2}+\boldsymbol{G}_{2} \otimes \boldsymbol{G}_{1}\right)-\alpha_{12}\left(\lambda_{1}^{-2} \mathbf{A}_{1}+\lambda_{2}^{-2} \mathbf{A}_{2}\right)\right], \\
& \frac{\partial \mathbf{C}}{\partial \alpha_{12}}=\lambda_{1} \lambda_{2}\left(\boldsymbol{G}^{1} \otimes \boldsymbol{G}^{2}+\boldsymbol{G}^{2} \otimes \boldsymbol{G}^{1}\right) .
\end{aligned}
$$

With the explicit form of the stress projection $\hat{\mathbb{P}}$ in hand theory of the constrained convected metric method can be finalized by interpreting the mechanical meaning of the introduced Lagrange multipliers $\left\{\varrho^{i}\right\}_{i=1}^{n}$. Further the projection can be characterized as orthogonal or oblique. Remarkably, the interpretation of the multipliers can be established without assuming any particular constitutive form of the material.

Proposition 3.8 (Determination of the Lagrange multipliers) The Lagrange multipliers are determined as,

$$
\varrho^{i}=\frac{1}{2}\left\langle\partial_{\phi_{i}} \mathbf{C}, \mathbf{S}\right\rangle, \quad i=1,2, \ldots, n .
$$

Proof The relation is obtained using the derived decomposition of the stress (3.30), the representation (3.10) for the workless stress and the duality (3.32). This proposition concludes the unique stress decomposition using the constrained convected metric approach.

The result deserves a pair of illustrations.

Example 1 (Incompressibility) Inserting the expression $\partial_{J} \mathbf{C}$ from Example 1 into (3.37) the Lagrange multiplier is determined as the mean hydrostatic stress,

$$
\varrho=\frac{1}{3} J^{-1}\langle\mathbf{C}, \mathbf{S}\rangle
$$


Example 2 (Two oblique plain extensibility constraints) Using the expression $(3.35)_{2}$ the Lagrange multipliers are determined by;

$$
\varrho^{\mathrm{F}}=\frac{1}{2}\left\langle\partial_{\lambda_{\mathrm{F}}} \mathbf{C}, \mathbf{S}\right\rangle, \quad \mathrm{F}=1,2
$$

For oblique constraints shear stresses are involved.

Example 3 (Angle preservation/unshearability) Using the expression (3.36) 3 the Lagrange multiplier for angle preservation in the 12-plane $(a=1$ and $b=2)$ becomes the associated shear stress;

$$
\varrho=\frac{1}{2}\left\langle\partial_{\alpha_{12}} \mathbf{C}, \mathbf{S}\right\rangle=\lambda_{1} \lambda_{2}\left\langle\mathbf{S} \boldsymbol{G}_{1}, \boldsymbol{G}_{2}\right\rangle
$$

The last task that needs to be investigated is whether the stress projection $\hat{\mathbb{P}}$ is self-adjoint or not. That is, if $\hat{\mathbb{P}}$ is an orthogonal or oblique projection. The complementary projection to $\hat{\mathbb{P}}, \mathbb{Q}:=\mathbb{I}-\hat{\mathbb{P}}$ will be a sum of component projections $\hat{\mathbb{Q}}^{i}$. The total projection will be selfadjoint if all the components are self-adjoint. To simplify matters a bit a single component of the complementary projection is studied,

$$
\hat{\mathbb{Q}}^{i}=\frac{\partial \phi_{i}}{\partial \mathbf{C}} \otimes \frac{\partial \mathbf{C}}{\partial \phi_{i}}
$$

For arbitrary tensors $\mathbf{S}, \mathbf{T} \in \operatorname{Sym}\left(T_{\boldsymbol{X}} \mathscr{P}_{0} \otimes T_{\boldsymbol{X}} \mathscr{P}_{0}\right)$ self-adjointness follows if, the left and right hand-sides below are equal, i.e., LHS $=$ RHS where:

$$
\begin{array}{ll}
\text { LHS: } & \left(\hat{\mathbb{Q}}^{i} \mathbf{T}, \mathbf{S}\right)_{\mathbf{C}}=\left\langle\partial_{\phi_{i}} \mathbf{C}, \mathbf{T}\right\rangle\left\langle\left[\partial_{\mathbf{C}} \phi_{i}\right]^{b}, \mathbf{S}\right\rangle, \\
\text { RHS: } & \left(\mathbf{T}, \hat{\mathbb{Q}}^{i} \mathbf{S}\right)_{\mathbf{C}}=\left\langle\partial_{\phi_{i}} \mathbf{C}, \mathbf{S}\right\rangle\left\langle\left[\partial_{\mathbf{C}} \phi_{i}\right]^{b}, \mathbf{T}\right\rangle,
\end{array}
$$

where $(*, *)_{\mathbf{C}}$ is the proper Lagrangian inner product (see Notation (N.2) and Appendix B), and where the definition $\mathbf{T}^{b}:=\mathbf{C T C}$ and the identity $\left\langle\mathbf{T}^{b}, \mathbf{S}\right\rangle=\left\langle\mathbf{S}^{b}, \mathbf{T}\right\rangle$ is used.

Proposition 3.9 (Sufficient condition for self-adjointness) A sufficient condition for $\hat{\mathbb{Q}}^{i}=\left[\hat{\mathbb{Q}}^{i}\right]^{*}$ is,

$$
\beta_{i} \partial_{\phi_{i}} \mathbf{C}=\left[\partial_{\mathbf{C}} \phi_{i}\right]^{b},
$$

where $\beta_{i}>0$. The multiplier $\beta_{i}$ can be determined taking the scalar product on both sides with $\partial_{\mathbf{C}} \phi_{i}$ and using (3.32);

$$
\beta_{i}=\left\langle\left[\partial_{\mathbf{C}} \phi_{i}\right]^{b}, \partial_{\mathbf{C}} \phi_{i}\right\rangle
$$

Proof The result is deduced by inspection of (3.42).

Example 1 (Incompressibility) For incompressibility, $n=1: \beta=3 / 4 J^{2}$,

$$
\beta \partial_{J}\left(J^{2 / 3} \overline{\mathbf{C}}\right)={ }^{1 / 2} J \mathbf{C}=\left[\partial_{\mathbf{C}} J\right]^{b} .
$$

The spherical projection in incompressibility is shown to be self-adjoint. Therefore, the stress decomposition in incompressibility is governed by an orthogonal projection. 
Example 2 (Two oblique plain extensibility constraints) For two oblique plain extensibility constraints, $n=2, \mathrm{~F}=1,2: \beta_{\mathrm{F}}=1 / 4 \lambda_{\mathrm{F}}^{2}$,

$$
\left.\begin{array}{l}
\beta_{\mathrm{F}} \partial_{\lambda_{\mathrm{F}}} \mathbf{C}=\frac{1}{2} \lambda_{\mathrm{F}}^{3} \boldsymbol{G}^{\mathrm{F}} \otimes{\underset{\sim}{\mathrm{F}}}^{\mathrm{F}}+\frac{1}{2} \alpha_{\mathrm{FH}} \lambda_{\mathrm{F}}^{2} \lambda_{\mathrm{H}} \operatorname{sym}\left\{{\underset{\sim}{\boldsymbol{G}}}^{\mathrm{F}} \otimes{\underset{\sim}{\boldsymbol{G}}}^{\mathrm{H}}\right\}+\frac{1}{2} \alpha_{\mathrm{F} 3} \lambda_{\mathrm{F}}^{2} \lambda_{3} \operatorname{sym}\left\{\underline{\sim}^{\mathrm{F}} \otimes{\underset{\sim}{G}}^{3}\right\} \\
{\left[\partial_{\mathrm{C}} \lambda_{\mathrm{F}}\right]^{\mathrm{b}}=\frac{1}{2} \lambda_{\mathrm{F}}^{-1} g_{\mathrm{JF}} \underline{\sim}^{\mathrm{J}} \otimes g_{\mathrm{KF}} \underline{\boldsymbol{G}}^{\mathrm{K}}, \quad(\operatorname{sum~J}, \mathrm{K})}
\end{array}\right\}
$$

where $\mathrm{H}=2,1$ for $\mathrm{F}=1,2$. The constrained spatial material/convected metric tensor $\hat{\mathbf{g}}(\boldsymbol{\varphi})$ will be oblique, in general. The stress decomposition induced by the constrained convected metric approach will therefore be governed by an oblique projection in case of two oblique simple extensibility constraints.

Example 3 (Angle preservation/unshearability) The sufficient condition for self-adjointness Proposition 3.9 for angle preservation in the 12-plane $(a=1$ and $b=2)$ is set up using expressions $(3.36)_{2,3}$ yielding $\beta=1 / 2\left(1-\alpha_{12}^{2}\right)^{2}$ and,

$$
\left.\begin{array}{rl}
\beta \partial_{\alpha_{12}} \mathbf{C}= & \frac{1}{2}\left(1-\alpha_{12}^{2}\right)^{2} \lambda_{1} \lambda_{2}\left(\underline{\sim}^{1} \otimes \underline{\sim}^{2}+\boldsymbol{G}^{2} \otimes \underline{\sim}^{1}\right) \\
{\left[\partial_{\mathbf{C}} \alpha_{12}\right]^{b}=} & \frac{1}{2}\left[\lambda_{1}^{-1} \lambda_{2}^{-1} g_{J 1} g_{K 2}\left(\underline{\sim}^{J} \otimes \boldsymbol{G}^{K}+\boldsymbol{G}^{K} \otimes \boldsymbol{G}^{J}\right)\right. \\
& \left.-\alpha_{12}\left(\lambda_{1}^{-2} g_{J 1} g_{K 1}+\lambda_{2}^{-2} g_{J 2} g_{K 2}\right) \boldsymbol{G}^{J} \otimes \underline{\sim}^{K}\right],
\end{array}\right\}
$$

for $J, K=1,2,3$. For $\alpha_{12}=0$,

$$
\left.\begin{array}{c}
\beta \partial_{\alpha_{12}} \mathbf{C}=\lambda_{1} \lambda_{2} \frac{1}{2}\left({\underset{\sim}{\boldsymbol{G}}}^{1} \otimes{\underset{\sim}{\boldsymbol{G}}}^{2}+{\underset{\sim}{\boldsymbol{G}}}^{2} \otimes \underline{\sim}^{1}\right) \\
{\left[\partial_{\mathbf{C}} \alpha_{12}\right]^{\mathrm{b}}=g_{J 1} g_{K 2} \lambda_{1}^{-1} \lambda_{2}^{-1} \frac{1}{2}\left({\underset{\sim}{\boldsymbol{G}}}^{J} \otimes{\underset{\sim}{G}}^{K}+{\underset{\sim}{\boldsymbol{G}}}^{K} \otimes{\underset{\sim}{\boldsymbol{G}}}^{J}\right),}
\end{array}\right\}
$$

for $J, K=1,2,3$. The induced stress projection is orthogonal, for an orthogonal spatial metric. Otherwise it is oblique.

Thus, it can be concluded that the constrained convected metric approach induced decomposition of the stress may be orthogonal or oblique. The outcome is case dependent. ${ }^{10}$

\section{Working Stress Determination; Hyperelasticity, Clausius-Planck Procedure}

In this section the consequences of the of simple internal constraints on the constitutive aspects are considered. The determination of the constitutive expression for the working stress induced by the constrained convected metric approach is outlined in a purely mechanical situation, using the Clausius-Planck procedure. For brevity and transparency the procedure is illustrated assuming a hyperelastic material response.

Proposition 4.1 (Lagrange multiplier/Clausius-Planck procedure) A twice time continuously differentiable strain energy function $\Psi(\mathbf{C}) \equiv \Psi(\hat{\mathbf{C}})$ with $\hat{\mathbf{C}} \in \mathscr{M} \subset \subset \operatorname{Sym}^{+}\left(T_{X}^{*} \mathscr{P}_{0} \otimes\right.$

\footnotetext{
${ }^{10}$ As opposed to the constraint manifold approach [7] which prescribes an orthogonal decomposition.
} 
$\left.T_{X}^{*} \mathscr{P}_{0}\right)$ (Definition 3.2) is assumed. Parametric dependence on structural tensors is suppressed for transparency. The strain energy is stated in the following augmented Lagrange multiplier form;

$$
\Psi\left(\mathbf{C},\left\{\varrho^{i}\right\}_{i=1}^{n}\right)=\Psi(\hat{\mathbf{C}})+\sum_{i=1}^{n} \varrho^{i} \hat{\phi}_{i}\left(\mathbf{C} ; \phi_{i}^{0}\right), \quad \text { for } \hat{\mathbf{C}}(\mathbf{C}) \in \mathscr{M}
$$

Inserting this ansatz into the Lagrangian form of the Clausius-Planck law,

$$
\left\langle\frac{1}{2} \dot{\mathbf{C}}, \mathbf{S}\right\rangle-\dot{\Psi} \geq 0
$$

yields the following expression for the total stress induced by the constrained convected metric approach;

$$
\mathbf{S}=\mathbf{S}_{\mathrm{R}}+\mathbf{S}_{\mathrm{A}}, \quad\left\{\begin{array}{l}
\mathbf{S}_{\mathrm{R}}=\sum_{i=1}^{n} 2 \varrho^{i} \frac{\partial \hat{\phi}_{i}}{\partial \mathbf{C}}, \quad \varrho^{i}=\frac{1}{2}\left\langle\partial_{\phi_{i}} \mathbf{C}, \mathbf{S}\right\rangle, \\
\mathbf{S}_{\mathrm{A}}=\hat{\mathbb{P}}\left[2 \frac{\partial \Psi(\hat{\mathbf{C}})}{\partial \hat{\mathbf{C}}}\right],
\end{array}\right.
$$

where $\hat{\mathbb{P}}$ is the stress projection, see Propositions 3.4 and 3.7.

Proof The result is obtained applying the celebrated Coleman and Noll procedure [2]. It states that the arguments of $\dot{\mathbf{C}}$ and $\dot{\varrho}^{i}$ must vanish independently. The argument of $\dot{\mathbf{C}}$ yields (4.3). The latter returns the imposed constraints, $\phi_{i}(\mathbf{C} ; \mathscr{S})=\phi_{i}^{0}$, for $i=1,2, \ldots, n$.

Remark 1 The strain energy function $\Psi(\hat{\mathbf{C}} ; \ldots)$ may model an isotropic or anisotropic hyperelastic material. The internal constraints will mask certain of its response functions in view of that $\hat{\mathbf{C}} \in \mathscr{M}$. For example, the third invariant $I_{3}(\hat{\mathbf{C}}):=\operatorname{det}\left[\mathbf{C G}^{-1}\right] \equiv 1$ for incompressibility and $I_{4}\left(\hat{\mathbf{C}} ; \mathbf{A}_{1}\right):=\left\langle\mathbf{C}, \mathbf{A}_{1}\right\rangle \equiv 1$ for a single internal constraint coinciding with the preferred direction given by $\mathbf{A}_{1}$, are trivial, respectively.

Remark 2 Since $\hat{\mathbb{P}}^{2}=\hat{\mathbb{P}}$ is idempotent one may write,

$$
\mathbf{S}_{\mathrm{A}}=\hat{\mathbb{P}}\left[2 \frac{\partial \Psi(\mathbf{C})}{\partial \mathbf{C}}\right], \quad \text { with } \Psi(\mathbf{C}) \equiv \Psi(\hat{\mathbf{C}}), \quad \hat{\mathbf{C}} \equiv \mathbf{C} \in \mathscr{M}
$$

by assumption. The implication of this form is two-fold: first, notably the argument within brackets is now the unconstrained form of a hyperelastic solid, second, the form underlines the fact that the constrained convected metric approach separates the kinematic aspect from the constitutive one. In other words, an unconstrained material may for example be subjected to a testing programme that keeps the stretch fixed in a given direction. It will respond essentially as if it was subject to an internal extensibility constraint. Recall that the projection $\hat{\mathbb{P}}^{*}:=\partial \hat{\mathbf{C}} / \partial \mathbf{C}$ is induced by the constructed $\hat{\mathbf{C}} \in \mathscr{M}$. Note that, it is not claimed that the constructed constrained Lagrangian convected metric tensor $\hat{\mathbf{C}}$ is unique. However, in view of the projection theorem (see Appendix D, and Oden and Demkowicz [32, Prop. 2.7.1, p. 172]) $\hat{\mathbb{P}}$ induces a unique decomposition of the hyperelastic stress response function $\partial \Psi(\mathbf{C}) / \partial \mathbf{C}$. Given the constraint manifold it determines the corresponding work performing stress. 
Remark 3 (Uniqueness of $\hat{\mathbf{F}}$ ) The Flory split [22] of the deformation gradient determines its unimodular form $\operatorname{det} \hat{\mathbf{F}} \equiv 1$ by isotropic scaling $\hat{\mathbf{F}}:=(\operatorname{det} \mathbf{F})^{-1 / 3} \mathbf{1 F}$ using the actual dilatation $\alpha \mathbf{1}, \alpha=(\operatorname{det} \mathbf{F})^{-1 / 3}$. Hence all components $\mathrm{F}_{A}^{k}$ are scaled. The unimodular form is determined uniquely.

Given a referential preferred unit direction $\hat{\boldsymbol{M}}_{1} \in \mathscr{P}_{0}$ the associated stretch $\alpha$ in the transplacement $\boldsymbol{\varphi}$ is calculated as $\alpha^{2}=\hat{\boldsymbol{M}}_{1} \cdot \mathbf{C} \hat{\boldsymbol{M}}_{1}$. Using isotropic scaling by $\alpha^{-1}$ yields $\hat{\mathbf{F}}_{1}=\alpha^{-1} \mathbf{1 F}$, such that, $\hat{\mathbf{F}}_{1} \hat{\boldsymbol{M}}_{1}=\alpha^{-1} \alpha \hat{\boldsymbol{m}}_{1}=\hat{\boldsymbol{m}}_{1}$, where $\left\|\hat{\boldsymbol{m}}_{1}\right\|=1$. Thus, $\hat{\mathbf{F}}_{1}$ is an admissible deformation gradient for $a$ simple inextensibility in the preferred direction $\hat{\boldsymbol{M}}_{1}$. The presented method in Sect. 3.2 yields a different admissible candidate $\hat{\mathbf{F}}_{2}=[\mathbf{1}+$ $\left.\left(\alpha^{-1}-1\right) \hat{\boldsymbol{m}}_{1} \otimes \hat{\sim}_{1}\right] \mathbf{F}$ such that $\hat{\mathbf{F}}_{2} \hat{\boldsymbol{M}}_{1}=\hat{\boldsymbol{m}}_{1}$. The two methods modify the volume ratio as, $\operatorname{det} \hat{\mathbf{F}}_{1}=\alpha^{-3} J$ and $\operatorname{det} \hat{\mathbf{F}}_{2}=\alpha^{-1} J$, where the determinant rules $\operatorname{det}[a \mathbf{T}]=a^{3} \operatorname{det} \mathbf{T}$ and $\operatorname{det}[b \mathbf{1}+c \boldsymbol{v} \otimes \boldsymbol{v}]=b^{2}(b+c)$ are used, respectively. Two comments are in place:

(1) The isotropic scaling changes the volume ratio by the factor $\alpha^{-3}$, while the advocated method scales the actual volume ratio by the factor $\alpha^{-1}$. Assume that the actual deformation gradient is a simple extension without lateral contraction in the preferred direction $\hat{\boldsymbol{M}}_{1}$. The presented construction method in Sect. 3.2 properly yields an identity mapping $\hat{\mathbf{F}}_{2}=\mathbf{I}$ and the right volume ratio $\operatorname{det}\left[\hat{\mathbf{F}}_{2}\right]=1$, while the isotropic scaling version yields an admissible but physically not acceptable result, $\hat{\mathbf{F}}_{1}=\alpha^{-1} \mathbf{I}+\left(1-\alpha^{-1}\right) \hat{\boldsymbol{m}}_{1} \otimes \hat{\boldsymbol{M}}_{1}$ and a wrong, non-trivial, modified volume ratio, $\operatorname{det}\left[\hat{\mathbf{F}}_{1}\right]=\alpha^{-2}\left[\alpha^{-1}+\left(1-\alpha^{-1}\right)\right]=\alpha^{-2}$.

(2) Further it is evident that the isotropic scaling method can in general only provide a candidate admissible deformation gradient for a case with a single constraint.

In conclusion, the presented method in Sect. 3.2 constructs an admissible deformation gradient which is physically acceptable. It has been demonstrated also for cases with two simultaneous constraints, see Example 2 and Remark 7. Here it is shown, that other constructed candidates may be members of the constraint manifold, but may not necessarily be physically acceptable.

\section{Extension to Nearly-Constrained Materials and Unconstrained Ones-The Generalised Metric Approach}

It would be preferred that an unconstrained material gradually transforms into the corresponding internally constrained material as its response in a certain mode of deformation declines when penalized by increasing the governing material parameter. The literature is however filled with separate constitutive postulates for uncoupled nearly-constrained materials. Again, near incompressibility relying on the celebrated Flory split of the deformation gradient $\mathbf{F}=J^{1 / 3} \overline{\mathbf{F}}$ provides a rich spectrum of such contributions. A prominent one is the pressure, dilatation, unimodular Cauchy-Green tensor, $\left(p, J, \overline{\mathbf{F}}^{*} \mathbf{g} \overline{\mathbf{F}}\right)$ three field formulation of the virtual work principle by Simo, Taylor and Pister [14]. This class of constitutive assumptions can be bypassed. It has no explicit part in the relaxation of constraints presented here. In this section a target component constraint manifold $\mathscr{M}_{\phi_{i}}$ is (on-off) relaxed. Or, when an additional physical quantity $\phi(\mathbf{C})$ becomes involved in a generalised constraint. 


\subsection{The Generalised Metric Approach}

In this subsection the procedure to construct a generalised Cauchy-Green/Lagrangianmetric tensor denoted $\tilde{\mathbf{C}}$ by modifying a given constrained Cauchy-Green/Lagrangianmetric tensor $\hat{\mathbf{C}}$ is presented, i.e., item $\mathscr{C}_{2}$ in the Introduction, the second main contributions of this work.

A question is whether the constrained convected metric $\hat{\mathbf{C}}$ can be relaxed keeping the same stress decomposition, and simultaneously transforming an associated Lagrange multiplier to a material response function. The answer is affirmative and the solution is strikingly simple, close and rational. The generalising steps are outlined very briefly, omitting technical details.

Definition 5.1 (Generalised constraint-variable substitution) A generalised constraint is equivalent with a variable substitution of the constrained quantity $\phi(\mathbf{C})$ by an, in the strong point-wise sense $(\doteq)$, equivalent auxiliary scalar independent strain-like variable $\tilde{\phi}$, replacing a kinematic quantity in one of the basic families $\mathscr{F}_{1}$ or $\mathscr{F}_{2}$, see Definition 3.1;

$$
\phi(\mathbf{C}(\varphi) ; \mathscr{S}) \doteq \tilde{\phi}(\boldsymbol{X}) .
$$

The generalised manifold is marked by a tilde sign $\tilde{\mathscr{M}}_{\phi}$;

$$
\tilde{\mathscr{M}}_{\phi}:=\left\{\mathbf{C}(\boldsymbol{\varphi}) \in \operatorname{Sym}^{+}\left(T_{X}^{*} \mathscr{P}_{0} \otimes T_{X}^{*} \mathscr{P}_{0}\right) \mid \phi(\mathbf{C}(\boldsymbol{\varphi}) ; \mathscr{S}) \doteq \tilde{\phi}(\boldsymbol{X})\right\} .
$$

Remark 4 It is noteworthy that the simple internal constraint (3.1) is replaced by a variable substitution (5.1). Formally, the parametric dependence on a referential value $\phi^{0}$ is replaced by an independent variable $\tilde{\phi}$. However, in the left hand-side $\varphi \in \mathscr{C}$ while the independent field in the right hand-side is in an auxiliary scalar point field. The matter is clear considering a weak formulation, see [31].

Remark 5 In computational mechanics, i.e., in finite precision arithmetic, a relaxed constraint manifold is often employed approximating the associated limiting one,

$$
\lim _{\tilde{\phi}_{i} \rightarrow \phi_{i}^{0}(\mathbf{G})} \tilde{\mathscr{M}}_{\phi_{i}}=\mathscr{M}_{\phi_{i}}, \quad i=1,2, \ldots, r .
$$

Not all constraints need to be relaxed, $r \leq n$. For transparency and simplicity, however, it is assumed henceforth that all constraints are relaxed, $r=n$ :

$$
\tilde{\mathscr{M}}:=\bigcap_{i=1}^{n} \tilde{\mathscr{M}}_{\phi_{i}} .
$$

Definition 5.2 (Generalised Lagrangian metric) A generalised Lagrangian metric tensor,

$$
\tilde{\mathbf{C}}=\tilde{\mathbf{C}}\left(\mathbf{C},\left\{\tilde{\phi}_{i}\right\}_{i=1}^{n}\right) \in \operatorname{Sym}^{+}\left(T_{X}^{*} \mathscr{P}_{0} \otimes T_{X}^{*} \mathscr{P}_{0}\right) \times \mathbb{R}^{r},
$$

satisfies by construction the variable substitutions in the generalised constraint manifold $\tilde{\mathscr{M}}$ (5.3) identically:

$$
\tilde{\mathbf{C}} \in \tilde{\mathscr{M}} .
$$


Each variable substitution is satisfied as an identity, point-wise for $\boldsymbol{X} \in \mathscr{P}_{0}$;

$$
\phi_{i}(\tilde{\mathbf{C}} ; \mathscr{S}) \equiv \tilde{\phi}_{i} \quad \Rightarrow \quad \tilde{\mathbf{C}} \equiv \mathbf{C}
$$

in the strong sense, where $\tilde{\mathbf{C}}$ should be understood as the value of the tensor function $\tilde{\mathbf{C}}\left(\mathbf{C}(\boldsymbol{\varphi}),\left\{\tilde{\phi}_{i}\right\}_{i=1}^{n} ; \mathscr{S}\right)$.

A mode of deformation which was extracted/annihilated from the spatial metric (Proposition 3.1) can be reinstated using the same mapping (3.13). The change of variable (5.1) can be performed. The following reinstatement mapping is introduced to that end;

$$
\tilde{\mathbf{f}}:=\prod_{i=1}^{r} \mathbf{f}\left(\tilde{\phi}_{i}\right) \in \operatorname{Lin}^{+}\left(T_{\varphi(X)} \mathscr{P} \otimes T_{\varphi(X)}^{*} \mathscr{P}\right) \times \mathbb{R}^{r}
$$

Proposition 5.1 (Preparation of a generalised Lagrangian metric) Given the actual generalised constraint manifold $\tilde{\mathscr{M}},(5.3)$, the constrained tangent map $\hat{\mathbf{F}}$ and the corresponding constrained spatial metric $\hat{\mathbf{g}}$, to be relaxed; the following composition defines the admissible generalised tangent map:

$$
\tilde{\mathbf{f}} \hat{\mathbf{F}}=: \tilde{\mathbf{F}} \quad \text { such that } \tilde{\mathbf{F}}^{*} \hat{\mathbf{g}} \tilde{\mathbf{F}} \in \tilde{\mathscr{M}}
$$

where the associated generalised spatial and Lagrangian metric tensors are identified;

$$
\tilde{\mathbf{C}}:=\mathbf{F}^{*} \tilde{\mathbf{g}} \mathbf{F} \quad \text { where } \tilde{\mathbf{g}}:=\tilde{\mathbf{f}}^{*} \hat{\mathbf{g}} \tilde{\mathbf{f}}
$$

Proof This proposition is proven in exactly the same way as Proposition 3.1, see Appendix C. The insertion mapping $\mathbf{f}(\tilde{\phi})$ is used instead of the annihilation mapping $\mathbf{f}^{-1}(\phi)$.

Proposition $5.2\left(\mathrm{~d}_{\mathrm{t}} \tilde{\mathbf{C}}\right.$ in the tangent set $\left.T_{\mathbf{C}} \tilde{\mathscr{M}}\right)$ The material time rate $\mathrm{d}_{\mathrm{t}} \tilde{\mathbf{C}}$ is in the tangent set to the generalised manifold, denoted $T_{\mathbf{C}} \tilde{\mathscr{M}}$;

$$
\left.\begin{array}{l}
\mathrm{d}_{\mathrm{t}} \tilde{\mathbf{C}} \in T_{\mathbf{C}} \tilde{\mathscr{M}}:=\bigcap_{i=1}^{n} T_{\mathbf{C}} \tilde{\mathscr{M}}_{\phi_{i}}, \\
T_{\mathbf{C}} \tilde{\mathscr{M}}_{\phi_{i}}:=\left\{\dot{\mathbf{C}} \in \operatorname{Sym}\left(T_{X}^{*} \mathscr{P}_{0} \otimes T_{X}^{*} \mathscr{P}_{0}\right), \quad \mathrm{d}_{\mathrm{t}} \tilde{\phi}_{i} \in \mathbb{R} \mid\left\langle\dot{\mathbf{C}}, \partial_{\mathbf{C}} \phi_{i}(\mathbf{C} ; \mathscr{S})\right\rangle \doteq \mathrm{d}_{\mathrm{t}} \tilde{\phi}_{i}\right\} .
\end{array}\right\}
$$

Proof The generalised convected Lagrangian metric $\tilde{\mathbf{C}} \in \tilde{\mathscr{M}}$, by $(5.5) \phi_{i}(\tilde{\mathbf{C}}) \equiv \tilde{\phi}_{i}$ for all variable substitutions $i$;

$$
\mathrm{d}_{\mathrm{t}} \phi_{i}(\tilde{\mathbf{C}})-\mathrm{d}_{\mathrm{t}} \tilde{\phi}_{i}=\left\langle\mathrm{d}_{\mathrm{t}} \tilde{\mathbf{C}}, \partial_{\tilde{\mathbf{C}}} \phi_{i}(\tilde{\mathbf{C}})\right\rangle-\mathrm{d}_{\mathrm{t}} \tilde{\phi}_{i} \equiv 0,
$$

for all variable substitutions $i$.

The equivalence (5.5) implies the rate equivalence,

$$
\mathrm{d}_{\mathrm{t}} \tilde{\mathbf{C}} \equiv \dot{\mathbf{C}}
$$

From the time rate of (5.1) and (5.9) the following identity it follows that,

$$
\partial_{\tilde{\mathbf{C}}} \phi(\tilde{\mathbf{C}})=\partial_{\mathbf{C}} \phi(\mathbf{C}) .
$$


Using (5.11) the time rate $\mathrm{d}_{\mathrm{t}} \tilde{\mathbf{C}}$ is expanded as,

$$
\mathrm{d}_{\mathrm{t}} \tilde{\mathbf{C}}=\underbrace{\left[\tilde{\mathbb{P}}^{*}+\sum_{i=1}^{n} \frac{\partial \tilde{\mathbf{C}}}{\partial \tilde{\phi}_{i}} \otimes \frac{\partial \phi_{i}}{\partial \mathbf{C}}\right]}_{=\mathbb{I}} \dot{\mathbf{C}}, \quad \tilde{\mathbb{P}}^{*}:=\frac{\partial \tilde{\mathbf{C}}}{\partial \mathbf{C}},
$$

where the under-braced conclusion is obtained in view of (5.10). Using (5.11) the duality (3.32) can readily be generalised to,

$$
\left\langle\partial_{\tilde{\phi}_{i}} \tilde{\mathbf{C}}, \partial_{\mathbf{C}} \phi_{j}\right\rangle=\delta_{j}^{i}, \quad i, j=1,2, \ldots, n
$$

Computing the partial derivative $\partial_{\tilde{\phi}_{i}} \hat{\phi}_{j}\left(\mathbf{C} ; \tilde{\phi}_{j}\right)$.

Proposition 5.3 (Generalised strain rate and stress projection) The generalised metric approach induces a projection of the strain rate;

$$
\tilde{\mathbb{P}}^{*}:=\frac{\partial \tilde{\mathbf{C}}}{\partial \mathbf{C}} \equiv \mathbb{I}-\sum_{i=1}^{n} \frac{\partial \tilde{\mathbf{C}}}{\partial \tilde{\phi}_{i}} \otimes \frac{\partial \phi_{i}}{\partial \mathbf{C}} .
$$

Idempotency $\left[\tilde{\mathbb{P}}^{*}\right]^{2}=\tilde{\mathbb{P}}^{*}$ follows using the generalised duality (5.13). The stress decomposition is obtained as the dual projection, $\tilde{\mathbb{P}}=\tilde{\mathbb{P}}^{* *}$ :

$$
\tilde{\mathbb{P}}:=\left[\frac{\partial \tilde{\mathbf{C}}}{\partial \mathbf{C}}\right]^{*} \equiv \mathbb{I}-\sum_{i=1}^{n} \frac{\partial \phi_{i}}{\partial \mathbf{C}} \otimes \frac{\partial \tilde{\mathbf{C}}}{\partial \tilde{\phi}_{i}} .
$$

Proof The identity (5.14) $)_{2}$ follows from (5.12) and (5.10).

Proposition 5.4 (Constraint relaxation-the generalised metric method)

1. Target constraints $\phi_{i}(\hat{\mathbf{C}})=\phi_{i}^{0}, i=1,2, \ldots, r$ (Definition 3.1) are changed to variable substitutions using Definition 5.1.

2. The generalised metric $\tilde{\mathbf{C}}$ is prepared according to the Proposition 5.1.

3. The Coleman and Noll procedure Proposition 4.1 is recalculated taking into consideration the new $r \leq n$ auxiliary kinematic variables $\left\{\tilde{\phi}_{i}\right\}_{i=1}^{r}$, i.e., $\mathrm{d}_{\mathrm{t}} \tilde{\phi}_{i} \neq 0$.

4. The stress decomposition is determined using the projection (5.15).

The r-relaxed components in $\mathbf{S}_{\mathrm{R}}$ are no longer reactions but constitutively determinate.

Proof There is nothing really to prove in this procedure. The outcome of Step 3 is stated in the next Proposition 5.5. Step 4 is given by Proposition 3.5 exchanging $\hat{\mathbb{P}}$ by $\tilde{\mathbb{P}}$.

To each auxiliary kinematic variable $\tilde{\phi}_{i}$ a work-conjugate material stress response function, denoted $\tilde{\varrho}^{i}$ is associated, which previously was the Lagrange multiplier, $\varrho^{i}$, see Definition 3.6. Their determination (Step 3 in Proposition 5.4) assuming hyperelasticity is outlined.

Proposition 5.5 (New stress response functions) In view of (4.3) $)_{3}$ and (4.4) the new stress response functions are determined as follows,

$$
\tilde{\varrho}^{i}=\left\langle\frac{1}{2} \frac{\partial \mathbf{C}}{\partial \phi_{i}}, 2 \frac{\partial \Psi(\mathbf{C})}{\partial \mathbf{C}}\right\rangle \equiv\left\langle\frac{1}{2} \frac{\partial \tilde{\mathbf{C}}}{\partial \tilde{\phi}_{i}}, 2 \frac{\partial \Psi(\tilde{\mathbf{C}})}{\partial \tilde{\mathbf{C}}}\right\rangle=\frac{\partial \Psi}{\partial \tilde{\phi}_{i}}, \quad i=1,2, \ldots, r,
$$


where $\tilde{\mathbf{C}} \equiv \mathbf{C} \in \tilde{\mathscr{M}}$. Here $\operatorname{dim} \tilde{\mathbf{C}}=\operatorname{dim} \mathbf{C}=6-(n-r)$ where $r$ is the number of relaxed constraints.

Proof The result relies on the variable substitution (5.1) which implies $\tilde{\mathbf{C}} \equiv \mathbf{C} \in \tilde{\mathscr{M}}$ which in turn implies $\Psi(\tilde{\mathbf{C}}) \equiv \Psi(\mathbf{C})$. The final result $(5.16)_{3}$ is understood folding the duality parings from right to left.

Example 1 (Incompressibility) Applying (5.16) to relaxed incompressibility and simplifying one then obtains the classic constitutive expression for the volumetric response function:

$$
\tilde{\varrho}=\left\langle\frac{1}{2} \partial_{J} \mathbf{C}, 2 \partial_{\mathbf{C}} \Psi\right\rangle=\partial_{J} \Psi .
$$

Example 2 (Two oblique plain extensibility constraints) Applying (5.16) relaxing two oblique plain extensibility constraints and simplifying one then obtains the constitutive expression for the fibre tension response functions:

$$
\tilde{\varrho}^{\mathrm{F}}=\left\langle\frac{1}{2} \partial_{\lambda_{\mathrm{F}}} \mathbf{C}, 2 \partial_{\mathbf{C}} \Psi\right\rangle=\partial_{\lambda_{\mathrm{F}}} \Psi, \quad \text { for } \mathrm{F}=1,2 .
$$

Example 3 (Angle preservation/unshearability) Relaxing an angle preservation constraint in the 12-plane ( $a=1$ and $b=2$ ) one obtains the constitutive expression for the associated shear stress,

$$
\tilde{\varrho}=\left\langle\frac{1}{2} \partial_{\alpha_{12}} \mathbf{C}, 2 \partial_{\mathbf{C}} \Psi\right\rangle=\partial_{\alpha_{12}} \Psi
$$

Remark 6 In view of the folding property of projections ${ }^{11}$ the $r$ relaxed responses can be removed from the decomposition in the strong point-wise sense. Considering a weak formulation it is however an advantage, or even the purpose, to keep the set of auxiliary variables $\left\{\tilde{\phi}_{i}, \tilde{\varrho}^{i}\right\}_{i=1}^{r}$ separate allowing a mixed formulation. The finite element realisation of a reinforcement of a material by two oblique strongly anisotropic fibre families is considered in the recently published work [31].

\section{Discussion}

The presented constrained convected metric approach to hyperelastic solids with simple internal constraints is coherent in essence with the classic theory Truesdell and Noll [1] and with the constraint manifold approach Podio-Guidugli and associates [4-6] and Carlson et al. [7]. From a historical point of view, the idea developed from two observations. The first was the view that the right Cauchy-Green tensor may be seen to be the referential Lagrangian convected metric tensor, obtained by pull-back of the spatial metric tensor, ${ }^{12}$ the second was that its unimodular version $\mathbf{F}^{*} \mathbf{g} \mathbf{F}=\overline{\mathbf{F}}^{*} \mathbf{g} \overline{\mathbf{F}}^{13}$ satisfies the incompressibility constraint identically, by construction. The employment of given preferred directions in the

\footnotetext{
${ }^{11} \mathbb{P}+\mathbb{Q}=\mathbb{I}$.

${ }^{12}$ Follows on using tensor algebra on manifolds $[12,13]$, where $\mathbf{C}: T_{\boldsymbol{X}} \mathscr{P}_{0} \rightarrow T_{\boldsymbol{X}}^{*} \mathscr{P}_{0}$, is metric like, while in classic continuum mechanics [1] it is introduced as $\mathbf{F}^{\top} \mathbf{F}: T_{\boldsymbol{X}} \mathscr{P}_{0} \rightarrow T_{\boldsymbol{X}} \mathscr{P}_{0}$ with no immediate connection to the spatial metric tensor.

${ }^{13}$ See the connection to the celebrated split of the deformation gradient $\mathbf{F}=J^{1 / 3} \mathbf{1} \overline{\mathbf{F}}$ due to Flory [22] used in the Simo, Taylor and Pister [14] three field formulation of the virtual work principle.
} 
sense of Spencer [16] constructing a special-purpose local-triad based convected parametrisation is a key element to handle especially multiple anisotropic constraints with oblique preferred directions, [31].

The principle of determinism for simple materials subject to internal constraints [1, Sect. 30, p.70] introduces an additive decomposition of the stress. Without a normalisation condition it is not unique. In the classic theory the normalisation condition is optional; it is suggested by example [1, Sect. 30, Eq. (30.17)] see also Spencer [16, p. 184]. It serves two purposes. First it is used to remove indeterminacies from the work-performing stress. Having done that, it enables the mechanical interpretation of the Lagrange multiplier(s), e.g. as the mean hydrostatic stress or as a uniaxial tension.

In the constraint manifold theory Carlson et al. [7] it is assumed that the work-performing stress is perpendicular to the workless stress. It must be emphasized that this is an assumption. To my best knowledge there is no generally accepted principle that prescribes it. One of the main results of this contribution is that the relation between the work-performing stress and the workless stress is determined by the constructed constrained Cauchy-Green tensor to be an element of the constraint manifold, starting from an arbitrary actual CauchyGreen tensor. It is found that these stresses are not always perpendicular. Sometimes they are oblique. Two examples with possible oblique stress projections (decompositions) are provided. Inextensibility in two oblique preferred directions and a preservation of the angle between two oblique preferred directions. The consequences are interesting and reaching beyond the scope of this work.

Further, it is noted that the direct sum decomposition of the Lagrangian stretch rate is induced by the fact it is an element of the tangent set to the constraint manifold. The direct sum decomposition of the total stress follows from duality. Remarkably, it can be completed formally without any reference to a particular material law, in line with the observation by Carlson et al. [7]. The determination of the actual work performing stress response evidently requires a material law. The Clausius-Planck procedure is used herein for that purpose. It is a restriction to the purely mechanical situation of the celebrated Coleman-Noll approach based on the Clausius-Duhem law, see Coleman and Noll [2]. The restriction to finite hyperelasticity is done purely for transparency.

The classic theory of internal constraints requires the Cauchy-Green tensor to be in the constraint manifold. Adding this requirement to the the set of admissible transplacements (Definition 3.2) shows that a trial solution is requested to satisfy each kinematic constraint point-wise. Further, the classic theory of internal constraints requires the time rate of the Cauchy-Green stretch to be in the tangent set associated with the constraint manifold, at the admissible strain. Adding this requirement to the the set of admissible variations (Definition 3.5) shows that a test function $\delta \varphi \cong \Delta \varphi=\dot{\varphi} \Delta t$ is requested to satisfy the first variation of each constraint, point-wise. From the computational point of view, this is very hard to realise. It is known that it is difficult to construct test functions in three-space which are exactly solenoidal point-wise. A closer look at equation (3.23) indicates that the use of the modified Cauchy-Green tensor (Definition 3.7) enables the use of standard test functions.

The generalisation of the method for unconstrained materials is almost trivial. It relies on the observation that the Lagrange multiplier method can be changed into a variable substitution. A constraint is transformed into a variable substitution, introducing auxiliary kinematic variables. In essence, the transformation yields a Hu-Washizu approach. Relaxing the constraints turns Lagrange multipliers into material response functions. The number of constraints is reduced accordingly. Point-wise they may be folded on account of the projection theorem in linear algebra [21, Th. 17.3]. The relaxed degrees of freedom may be 
kept providing a basis together with the fully constrained ones for a mixed formulation. This possibility is exercised in the recently published work [31]. It should be noted that the compositions employed to annihilate and reinstate deformation modes are performed in the spatial configuration, without the introduction of an intermediate configuration, as opposed to other celebrated multiplicative splits of the deformation gradient, e.g. the one used for elasto-plasticity due to Lee [23].

Finally, the presentation of the constrained convected metric method is confined to simple or composed elementary constraints concerning compressibility, extensibility in a given preferred direction, or to the change of the angle between a pair of preferred directions. More general simple internal constraints, like for example the Ericksen constraint, $\left\langle\mathbf{C}, \mathbf{G}^{-1}\right\rangle=3$, fall outside the scope of this work.

\section{Summary, Conclusion and Outlook}

The purely mechanical consequences of imposing simple kinematic internal constraints are discussed. The constraint manifold restricting the set of admissible transplacements is formed. A Lagrangian setting using the reduced, objective, form of a constraint in terms of the right Cauchy-Green stretch tensor is employed. Starting from an arbitrary CauchyGreen tensor a modified Cauchy-Green tensor is constructed that satisfies all the subsidiary conditions in the constraint manifold as identities. It is shown that the time rate of a subsidiary condition written in terms of the modified Cauchy-Green tensor induces a projection. It defines the admissible Lagrangian strain rate as an element in the tangent set to the constraint manifold. By duality it defines a corresponding stress decomposition. It defines a work performing stress. A duality condition defining the complementarity between the workless part of the stress and the work performing part is identified. It also governs the determination of the Lagrange multipliers in terms of the total stress. The stress decomposition need not be orthogonal. It may be oblique. The sufficient condition for orthogonality (self-adjointness) is derived. Examples of oblique decompositions are provided. The determination of the work performing constitutive stress response function is exemplified using the Coleman and Noll procedure [2] based on the Clausius-Planck law for hyperelasticity. The theory and procedure for the modification of an arbitrary Cauchy-Green tensor such that it satisfies all constraints point-wise for an actual transplacement extends the classic theory [1] and the constraint manifold theory [7], remaining coherent with the classic foundations.

The theory and procedure for the relaxation of constraints seamlessly extending to unconstrained materials is shown. It gives rise to the so-called generalised metric method. A unified theory for a number of verified finite element applications [14, 27-31] is presented. Possible extensions are indicated, for example, formulation of a preservation of the angle between two families of material line elements alone or in combination with inextensibility or incompressibility. Extensions to non-isothermal conditions. Consideration of other not so elementary constraints, for example the Ericksen constraint etc.

Acknowledgement Open access funding provided by Swedish Defence Research Agency. I thank Prof. Waldemar Rachowicz, Institute of Computer Science, Cracow University of Technology, Pl 31-155 Cracow, Poland, wrachowicz@pk.edu.pl, who provided insight, expertise and suggestions that greatly assisted the research.

Publisher's Note Springer Nature remains neutral with regard to jurisdictional claims in published maps and institutional affiliations. 
Open Access This article is distributed under the terms of the Creative Commons Attribution 4.0 International License (http://creativecommons.org/licenses/by/4.0/), which permits unrestricted use, distribution, and reproduction in any medium, provided you give appropriate credit to the original author(s) and the source, provide a link to the Creative Commons license, and indicate if changes were made.

\section{Appendix A: Notation}

Vectors are denoted by boldface italic lower and upper case letters, e.g. $\boldsymbol{a}, \boldsymbol{b}, \boldsymbol{c}$ and $\boldsymbol{A}, \boldsymbol{B}, \boldsymbol{C}$ respectively. Linear forms or co-vectors are denoted with an under-set tilde, e.g. $\underset{\sim}{\boldsymbol{a}}, \underset{\sim}{\boldsymbol{b}}, \underset{\sim}{\boldsymbol{c}}$ and $\underset{\sim}{\boldsymbol{A}}, \underset{\sim}{\boldsymbol{B}}, \underset{\sim}{\boldsymbol{C}}$ respectively. Second order tensors are denoted by boldface Roman or Greek lower and upper case letters, e.g. $\boldsymbol{\tau}, \mathbf{a}, \mathbf{g}$ and $\mathbf{S}, \mathbf{A}, \mathbf{G}$. Fourth order tensors are denoted by blackboard bold lower and upper case letters, e.g. $\mathbb{p}$ and $\hat{\mathbb{P}}$ respectively. In particular, $\mathbb{I}$ denotes the fourth order identity mapping, i.e., $\mathbb{I} \mathbf{T}=\mathbf{T}$ for all $\mathbf{T}$. The contraction, dualitypairing, of co-vectors with vectors on a given vector space $\mathscr{V}=(\mathscr{F} ; \mathbb{R})$, is denoted as,

$$
\langle\bullet, \bullet\rangle_{\mathscr{V}}: \mathscr{V}^{*} \times \mathscr{V} \rightarrow \mathbb{R}, \quad(\underset{\sim}{\boldsymbol{B}}, \boldsymbol{A}) \mapsto\langle\underset{\sim}{\boldsymbol{B}}, \boldsymbol{A}\rangle_{\mathscr{V}} \in \mathbb{R}
$$

where for vectors $\mathscr{F}=\mathbb{R}^{3}$. The same bracket notation $\left(\langle\bullet, \bullet\rangle_{\mathscr{V}}\right)$ is used for the double contraction of compatible second order tensors defined on $\mathscr{F}=\mathbb{R}^{3} \otimes \mathbb{R}^{3}$ and on $\mathscr{F}=\operatorname{sym}\left\{\mathbb{R}^{3} \otimes \mathbb{R}^{3}\right\}$ respectively. Whenever there is no ambiguity to which vector space the contraction is referred to, the subscript index of $\langle\bullet, \bullet\rangle_{\mathscr{V}}$ will be suppressed. Further, the space of linear transformations $\operatorname{Lin}(\mathscr{V} ; \mathscr{W})$ is identified $(\cong)$ with the tensor space $\mathscr{W} \otimes \mathscr{V}^{*}$, i.e.,

$$
\operatorname{Lin}(\mathscr{V} ; \mathscr{W}) \cong \mathscr{W} \otimes \mathscr{V}^{*} .
$$

Furthermore, the inner product or dot product between a pair of vectors $(\boldsymbol{a}, \boldsymbol{b})$ on a metric space $(\mathscr{V}, \mathbf{g})$, with metric tensor $\mathbf{g}: \mathscr{V} \rightarrow \mathscr{V}^{*}$ is defined by,

$$
(\cdot, \cdot)_{\mathrm{g}}: \mathscr{V} \times \mathscr{V} \rightarrow \mathbb{R}, \quad(\boldsymbol{a}, \boldsymbol{b}) \mapsto(\boldsymbol{a}, \boldsymbol{b})_{\mathbf{g}}:=\langle\mathbf{g} \boldsymbol{a}, \boldsymbol{b}\rangle_{\mathscr{V}},
$$

where the metric $\mathbf{g}$ maps a vector into its associated linear form in the dual space, $\mathbf{g} \boldsymbol{a}=: \underset{\sim}{\boldsymbol{a}} \in \mathscr{V}^{*}$. In the literature the associated linear form $\underset{\sim}{\boldsymbol{a}}$ is sometimes denoted $\boldsymbol{a}^{b}$. When the basic metric on $\mathscr{V}$ is used, the notation is simplified by dropping the subscript, e.g. $(\boldsymbol{a}, \boldsymbol{b})_{\mathrm{g}}=(\boldsymbol{a}, \boldsymbol{b})$. When the inner product between $\boldsymbol{a}$ and $\boldsymbol{b}$ is computed using the Euclidean dot-product the standard notation, $\boldsymbol{a} \cdot \boldsymbol{b}$, is used. $T_{\boldsymbol{X}} \mathscr{P}_{0}$ and $T_{\boldsymbol{x}} \mathscr{P}$ will denote tangent vector spaces to Euclidean referential and spatial point manifolds $\mathscr{P}_{0}$ and $\mathscr{P}$, respectively. Finally, material time-differentiation is denoted by an over-dot $\left({ }^{\circ}\right)$. Finally, the norm of a vector $\boldsymbol{a}$ is denoted $\|\boldsymbol{a}\|:=\sqrt{(\boldsymbol{a}, \boldsymbol{a})}$.

\section{Appendix B: The Proper Lagrangian Inner Product}

The inner product of vectors $\boldsymbol{P}, \boldsymbol{R} \in\left(T_{\boldsymbol{X}} \mathscr{P}_{0}, \mathbf{C}\right)$ using the Lagrangian metric tensor $\mathbf{C}$; $(\boldsymbol{P}, \boldsymbol{R})_{\mathbf{C}}:=\langle\mathbf{C P}, \boldsymbol{R}\rangle_{T_{\boldsymbol{X}} \mathscr{P}_{0}}$ provides the proper referential inner product to the standard spatial inner product of the vectors $\boldsymbol{p}=\mathbf{F} \boldsymbol{P} \in T_{\varphi(X)} \mathscr{P}$ and $\boldsymbol{r}=\mathbf{F} \boldsymbol{R} \in T_{\varphi(X)} \mathscr{P}$ in the defor-

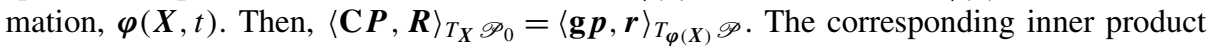
$(\mathbf{T}, \mathbf{S})_{\mathbf{C}}$ between tensors $\mathbf{T}, \mathbf{S} \in T_{X} \mathscr{P}_{0} \otimes T_{X} \mathscr{P}_{0}$ is used henceforth. Further, $\boldsymbol{P}^{\mathrm{b}}:=\mathbf{C P} \in$ $T_{X}^{*} \mathscr{P}_{0}$ is the linear form associated with $\boldsymbol{P} \in T_{X} \mathscr{P}_{0}$, using the metric $\mathbf{C}$, and $\mathbf{T}^{\mathrm{b}}:=\mathbf{C T C} \in$ $T_{X}^{*} \mathscr{P}_{0} \otimes T_{X}^{*} \mathscr{P}_{0}$ denotes the associated tensor corresponding to $\mathbf{T} \in T_{X} \mathscr{P}_{0} \otimes T_{X} \mathscr{P}_{0}$. 


\section{Appendix C: Proof of Proposition 3.1}

\section{C.1 Incompressibility}

Given the actual spatial metric tensor $\mathbf{g}$, the deformation gradient $\mathbf{F}$, the constraint manifold $\mathscr{M}_{\text {inc }}=\left\{\mathbf{C}=\mathbf{F}^{*} \mathbf{g F} \mid \operatorname{det}\left[\mathbf{C G}^{-1}\right]-1=0\right\}$ and the spatial bijective mapping,

$$
\left.\begin{array}{l}
\mathbf{f}(J): T_{\varphi(X)} \mathscr{P} \rightarrow T_{\varphi(X)} \mathscr{P}, \\
\mathbf{f}(J):=J^{1 / 3} \mathbf{1}, \quad J=\operatorname{det} \mathbf{F},
\end{array}\right\}
$$

where $\mathbf{1} w=w$ is the identity mapping on $T_{\varphi(X)} \mathscr{P}$. The dilatation in the actual deformation is annihilated point-wise computing the unimodular deformation gradient, $\hat{\mathbf{F}}=\mathbf{f}^{-1}(J) \mathbf{F}=$ $(\operatorname{det} \mathbf{F})^{-1 / 3} \mathbf{F}$, i.e., $\operatorname{det} \hat{\mathbf{F}}=1$. Compute the unimodular right Cauchy-Green stretch tensor (Lagrangian metric tensor), $\hat{\mathbf{C}}=\hat{\mathbf{F}}^{*} \mathbf{g} \hat{\mathbf{F}}=\mathbf{F}^{*} \hat{\mathbf{g F}}$, where $\hat{\mathbf{g}}:=\mathbf{f}^{-*} \mathbf{g f}^{-1}$. By the product rule $\operatorname{det}[\mathbf{A B C}]=\operatorname{det} \mathbf{A} \operatorname{det} \mathbf{B} \operatorname{det} \mathbf{C}, \operatorname{det} \hat{\mathbf{C}}=J^{-1} J^{2} J^{-1}=1$, which proves the required unimodularity of the constrained Lagrangian metric tensor, $\hat{\mathbf{C}} \in \mathscr{M}_{\text {inc }}$.

\section{C.2 Inextensibility}

The constraint manifold for a simple inextensibility in the referential unit direction $\boldsymbol{D}$ is defined by,

$$
\mathscr{M}_{\text {iext }}=\left\{\mathbf{C}=\mathbf{F}^{*} \mathbf{g F} \mid\|\boldsymbol{D}\|_{\mathbf{C}}-1=0\right\} .
$$

The spatial bijective uniaxial extension mapping for this case is,

$$
\left.\begin{array}{l}
\mathbf{f}(\lambda ; \boldsymbol{d}): T_{\varphi(\boldsymbol{X})} \mathscr{P} \rightarrow T_{\varphi(\boldsymbol{X})} \mathscr{P}, \\
\mathbf{f}(\lambda ; \boldsymbol{d}):=\mathbf{1}+(\lambda-1) \boldsymbol{d} \otimes \underset{\sim}{\boldsymbol{d}}, \quad \lambda=(\boldsymbol{d}, \mathbf{F} \boldsymbol{D})_{\mathbf{g}},
\end{array}\right\}
$$

where $\mathbf{F} \boldsymbol{D}=\lambda \boldsymbol{d},\|\boldsymbol{d}\|=1,\langle\underset{\sim}{\boldsymbol{d}}, \boldsymbol{d}\rangle=1$. The stretch $\lambda$ is annihilated point-wise in the spatial unit direction $\boldsymbol{d}$ computing the simply inextensible deformation gradient $\hat{\mathbf{F}}$ by the following composition,

$$
\left.\begin{array}{l}
\hat{\mathbf{F}}:=\mathbf{f}^{-1} \mathbf{F}=\left[\mathbf{1}+\left(\lambda^{-1}-1\right) \boldsymbol{d} \otimes \underset{\sim}{\boldsymbol{d}}\right] \mathbf{F}, \\
\hat{\mathbf{F}} \boldsymbol{D}=\lambda \boldsymbol{d}-\lambda \boldsymbol{d}+\lambda^{-1} \lambda \boldsymbol{d}=1 \boldsymbol{d} .
\end{array}\right\}
$$

The simply inextensible right Cauchy-Green stretch tensor (Lagrangian metric tensor) is computed as, $\hat{\mathbf{C}}=\hat{\mathbf{F}}^{*} \mathbf{g} \hat{\mathbf{F}}$. Evaluating the length of the material referential material line element in terms of the Lagrangian inextensible metric $\hat{\mathbf{C}}$, and using the expressions (C.4) yields,

$$
\|\boldsymbol{D}\|_{\hat{\mathbf{c}}}=\|\hat{\mathbf{F}} \boldsymbol{D}\|_{\mathrm{g}}=\|\boldsymbol{d}\|_{\mathrm{g}}=1 .
$$

Inserting (C.5) into (C.2) shows that $\hat{\mathbf{C}} \in \mathscr{M}_{\text {iext }}$, which completes the proof.

\section{C.3 Preservation of Angle}

The constraint manifold for the preservation of the angle between two referential unit directions $\boldsymbol{D}$ and $\boldsymbol{E}$ with mutual direction cosine $\alpha_{(\boldsymbol{D E})}^{0}:=(\boldsymbol{E}, \boldsymbol{D})$ is,

$$
\mathscr{M}_{\text {insh }}=\left\{\mathbf{C}=\mathbf{F}^{*} \mathbf{g F} \mid \alpha_{(\boldsymbol{D E})}-\alpha_{(\boldsymbol{D E})}^{0}=0\right\},
$$


where $\alpha_{(\boldsymbol{D E})}:=\lambda_{(\boldsymbol{D})}^{-1} \lambda_{(\boldsymbol{E})}^{-1}(\boldsymbol{E}, \boldsymbol{D})_{\mathbf{C}}$ is the Lagrangian expression for the corresponding spatial direction cosine, wherein $\lambda_{(\boldsymbol{D})}:=\|\boldsymbol{D}\|_{\mathbf{C}}$ and $\lambda_{(\boldsymbol{E})}:=\|\boldsymbol{E}\|_{\mathbf{C}}$ are the stretches in these directions. Using spatial unit direction vectors $\boldsymbol{d}:=\lambda_{(\boldsymbol{D})}^{-1} \mathbf{F} \boldsymbol{D},|\boldsymbol{d}|=1$ and $\boldsymbol{e}:=\lambda_{(\boldsymbol{E})}^{-1} \mathbf{F} \boldsymbol{E},\|\boldsymbol{e}\|=1$, the spatial bijective simple shear-like mapping for this case is given by,

$$
\left.\begin{array}{l}
\mathbf{f}(\gamma ; \boldsymbol{d}, \boldsymbol{e}): T_{\varphi(X)} \mathscr{P} \rightarrow T_{\varphi(\boldsymbol{X})} \mathscr{P}, \\
\mathbf{f}(\gamma ; \boldsymbol{d}, \boldsymbol{e}):=\mathbf{1}+\gamma \boldsymbol{d} \otimes \underset{\sim}{\boldsymbol{e}}, \quad \gamma:=\alpha_{(\boldsymbol{D E})}-\alpha_{(\boldsymbol{D E})}^{0},
\end{array}\right\}
$$

where $\langle\underset{\sim}{\boldsymbol{d}}, \boldsymbol{d}\rangle=1,\langle\underset{\sim}{\boldsymbol{e}}, \boldsymbol{e}\rangle=1$ and $\langle\underset{\sim}{\boldsymbol{d}}, \boldsymbol{e}\rangle=0$. The shear is annihilated point-wise by the following composition,

$$
\begin{gathered}
\hat{\mathbf{F}}=\mathbf{f}^{-1} \mathbf{F}=[\mathbf{1}-\gamma \boldsymbol{d} \otimes \underset{\sim}{\boldsymbol{e}}] \mathbf{F}, \\
\hat{\mathbf{F}} \boldsymbol{D}=\lambda_{(D)} \boldsymbol{d}-\gamma \lambda_{(D)}\langle\underset{\sim}{\boldsymbol{e}}, \boldsymbol{d}\rangle \boldsymbol{d}=\lambda_{(D)} \boldsymbol{d}, \\
\hat{\mathbf{F}} \boldsymbol{E}=\lambda_{(\boldsymbol{E})} \boldsymbol{e}-\gamma \lambda_{(E)}\langle\underset{\sim}{\boldsymbol{e}}, \boldsymbol{e}\rangle \boldsymbol{d}=\lambda_{(E)}(\boldsymbol{e}-\gamma \boldsymbol{d}) .
\end{gathered}
$$

The right Cauchy-Green stretch tensor (Lagrangian metric tensor) for a simple preservation of angle is computed as, $\hat{\mathbf{C}}=\hat{\mathbf{F}}^{*} \mathbf{g} \hat{\mathbf{F}}=\mathbf{F}^{*} \hat{\mathbf{g F}}$. Evaluating the inner product between $\boldsymbol{E}$ and $\boldsymbol{D}$ in terms of the Lagrangian inshearable metric $\hat{\mathbf{C}}$, and using the expressions (C.8) yields,

$$
\begin{aligned}
(\boldsymbol{E}, \boldsymbol{D})_{\hat{\mathbf{C}}} & =\langle\hat{\mathbf{C}} \boldsymbol{E}, \boldsymbol{D}\rangle=(\hat{\mathbf{F}} \boldsymbol{E}, \hat{\mathbf{F}} \boldsymbol{D})_{\mathrm{g}}=\lambda_{(E)} \lambda_{(D)}([\boldsymbol{e}-\gamma \boldsymbol{d}], \boldsymbol{d})_{\mathrm{g}} \\
& =\lambda_{(E)} \lambda_{(D)}\left(\alpha_{(D E)}-\gamma\right)=\lambda_{(E)} \lambda_{(D)} \alpha_{(D E)}^{0}
\end{aligned}
$$

Inserting (C.9) in (C.6) shows that $\hat{\mathbf{C}} \in \mathscr{M}_{\text {insh }}$, which completes the proof.

Remark 7 The simultaneous annihilation of a dilatation and a volume preserving stretch in the referential direction $\boldsymbol{D}$ in the deformation $\varphi(X, t)$ with deformation gradient $\mathbf{F}=\operatorname{Grad} \varphi$ is performed using a simple uniaxial extension with a lateral contraction, confer (C.3). The annihilation mapping used is;

$$
\left.\begin{array}{c}
\mathbf{f}(\lambda ; \boldsymbol{d}, \ell): T_{\varphi(X)} \mathscr{P} \rightarrow T_{\varphi(X)} \mathscr{P}, \\
\mathbf{f}(\lambda ; \boldsymbol{d}, \ell):=\ell \lambda(\mathbf{1}-\boldsymbol{d} \otimes \underset{\sim}{\boldsymbol{d}})+\lambda \boldsymbol{d} \otimes \underset{\sim}{\boldsymbol{d}}, \quad \operatorname{det} \mathbf{f}=\ell^{3} \lambda^{2},
\end{array}\right\}
$$

where $\ell=\ell(\lambda)>0$. It is noted that for $\ell=\lambda^{-1}$ the linear transformation $\mathbf{f}$ is purely uniaxial, while for $\ell=\lambda^{-2 / 3}$ it is volume preserving, $\operatorname{det} \mathbf{f}=1$, with an adjustment in the perpendicular direction $\mathbf{1}-\boldsymbol{d} \otimes \underset{\sim}{\boldsymbol{d}}$. Fixing $\ell=\lambda^{-2 / 3}$ it can be decomposed into a pure dilatation and a unimodular simple extension with lateral contraction,

$$
\mathbf{f}(\bar{\lambda}, J ; \boldsymbol{d})=\delta(J) \overline{\mathbf{f}}(\bar{\lambda} ; \boldsymbol{d}),\left\{\begin{array}{c}
\boldsymbol{\delta}(J):=J^{1 / 3} \mathbf{1}, \\
\overline{\mathbf{f}}(\bar{\lambda} ; \boldsymbol{d}):=\bar{\lambda}^{-1 / 2}(\mathbf{1}-\boldsymbol{d} \otimes \underset{\sim}{\boldsymbol{d}})+\bar{\lambda} \boldsymbol{d} \otimes \underset{\sim}{\boldsymbol{d}}, \quad \operatorname{det} \overline{\mathbf{f}} \equiv 1,
\end{array}\right.
$$

where $J=\operatorname{det} \mathbf{F}$ is the actual dilatation, and $\bar{\lambda}=\langle\underset{\sim}{\boldsymbol{d}}, \overline{\mathbf{F}} \boldsymbol{D}\rangle$ is the actual volume preserving stretch in the referential direction $\boldsymbol{D}$ defined as $\bar{\lambda}:=J^{-1 / 3} \lambda$ and where $\overline{\mathbf{F}}:=J^{1 / 3} \mathbf{F}$ is the actual unimodular deformation gradient. 


\section{Appendix D: The Projection Theorem}

If linear transformation $\mathbf{P}: \mathscr{V} \rightarrow \mathscr{V}$ is a projection, then

$$
\mathscr{V}=\mathrm{R}(\mathbf{P}) \oplus \mathrm{K}(\mathbf{P}),
$$

where $\mathrm{R}(\mathbf{P})$ and $\mathrm{K}(\mathbf{P})$ denote the range and the kernel of $\mathbf{P}$.

Proof Let $\boldsymbol{v}$ be an arbitrary vector in $\mathscr{V}$. Let,

$$
w=v-\mathbf{P} v
$$

Then on account of $\mathbf{P}^{2}=\mathbf{P}, \mathbf{P} \boldsymbol{w}=\boldsymbol{v}-\mathbf{P}(\mathbf{P} \boldsymbol{v})=\mathbf{P} \boldsymbol{v}-\mathbf{P} \boldsymbol{v}=\mathbf{0}$. Thus, $\boldsymbol{w} \in \mathrm{K}(\mathbf{P})$. Since $\mathbf{P} \boldsymbol{v} \in$ $\mathrm{R}(\mathbf{P})$, (D.2) implies that,

$$
\mathscr{V}=\mathrm{R}(\mathbf{P})+\mathrm{K}(\mathbf{P}) .
$$

To show that $\mathrm{R}(\mathbf{P}) \cap \mathrm{K}(\mathbf{P})=\{\mathbf{0}\}$, let $\boldsymbol{u} \in \mathrm{R}(\mathbf{P}) \cap \mathrm{K}(\mathbf{P})$. Then, since $\boldsymbol{u} \in \mathrm{R}(\mathbf{P})$ for some $\boldsymbol{v} \in \mathscr{V}$, $\boldsymbol{u}=\mathbf{P} \boldsymbol{v}$. But since, $\boldsymbol{u}$ is also in $\mathrm{K}(\mathbf{P})$,

$$
\mathbf{0}=\mathbf{P} u=\mathbf{P}(\mathbf{P} v)=\mathbf{P} v=u,
$$

which completes the proof.

\section{References}

1. Truesdell, C., Noll, W.: The Non-linear Field Theories of Mechanics, vol. 3. Springer, Berlin (1965)

2. Coleman, B.D., Noll, W.: The thermodynamics of elastic materials with heat conduction and viscosity. Arch. Ration. Mech. Anal. 13(1), 167-178 (1963)

3. Podio-Guidugli, P.: Constrained elasticity. Rend. Mat. Accad. Lincei 9(1), 341-350 (1990)

4. Podio-Guidugli, P., Vianello, M.: Constraint manifolds for isotropic solids. Arch. Ration. Mech. Anal. 105(2), 105-121 (1989)

5. Gurtin, M.E., Podio-Guidugli, P.: The thermodynamics of constrained materials. Arch. Ration. Mech. Anal. 51(3), 192-208 (1973)

6. Vianello, M.: Internal constraints in finite elasticity: manifolds or not. J. Elast. 114, 197-211 (2014)

7. Carlson, D.E., Fried, E., Tortorelli, D.A.: Geometrically-based consequences of internal constraints. J. Elast. 70, 1001-1009 (2003)

8. Bertram, A.: Elasticity and Plasticity of Large Deformations: An Introduction. Springer, Berlin (2008)

9. Bertram, A., Glüge, R.: Gradient materials with internal constraints. Math. Mech. Complex Syst. 4(1), $1-15$ (2016)

10. Negahban, M.: Single and multiple material constraints in thermoelasticity. Math. Mech. Solids 12(6), 623-664 (2007). https://doi.org/10.1177/1081286506067092

11. Steigmann, D.J.: Finite Elasticity Theory. Oxford University Press, Oxford (2017)

12. Marsden, J.E., Hughes, T.J.R.: Mathematical Foundations of Elasticity. Prentice Hall, New York (1983)

13. Stumpf, H., Hoppe, U.: The application of tensor algebra on manifolds to nonlinear continuum mechanics—invited survey article. Z. Angew. Math. Mech. 77(5), 327-339 (1997)

14. Simo, J.C., Taylor, R.L., Pister, K.S.: Variational and projection methods for the volume constraint in finite deformation elasto-plasticity. Comput. Methods Appl. Mech. Eng. 51, 177-208 (1985)

15. Green, A.E., Zerna, W.: Theoretical Elasticity. Oxford University Press, Oxford (1954)

16. Spencer, A.J.M.: Continuum Theory of the Mechanics of Fibre-Reinforced Composites. Springer, New York (1984)

17. Boehler, J. (ed.): Applications of Tensor Functions in Solid Mechanics. CISM Courses and Lectures, vol. 292. Springer, Berlin (1987)

18. Zhang, J., Rychlewski, J.: Structural tensors for anisotropic solids. Arch. Mech. 42, $267-277$ (1990)

19. Day, W.A.: The Thermodynamics of Simple Materials with Fading Memory. Springer, Berlin (1972). ISBN-13: 978-3-642-65320-9 
20. Noll, W.: The Foundations of Mechanics and Thermodynamics. Springer, Berlin (1974). Selected papers by W. Noll

21. Bowen, R.M., Wang, C.-C.: Introduction to Vectors and Tensors, Volumes 1 and 2. Plenum Press, New York (1976)

22. Flory, R.J.: Thermodynamic relations for highly elastic materials. Trans. Faraday Soc. 57, 829-838 (1961)

23. Lee, E.H.: Elastic-plastic deformation at finite strains. J. Appl. Mech. 36(1), 1-6 (1969). https://doi.org/ $10.1115 / 1.3564580$

24. Simo, J.C., Hughes, T.J.R.: Computational Inelasticity. Interdisciplinary Applied Mathematics, vol. 7. Springer, New York (1998)

25. Valanis, K.C.: The concept of physical metric in thermodynamics. Acta Mech. 113, 169-184 (1995)

26. Valanis, K.C., Panoskaltsis, V.P.: Material metric, connectivity and dislocations in continua. Acta Mech. 175, 77-103 (2005)

27. Zdunek, A., Rachowicz, W., Eriksson, T.: A novel computational formulation for nearly incompressible and nearly inextensible finite hyperelasticity. Comput. Methods Appl. Mech. Eng. 281, 220-249 (2014). https://doi.org/10.1016/j.cma.2014.08.008

28. Zdunek, A., Rachowicz, W., Eriksson, T.: A five-field finite element formulation for nearly inextensible and nearly incompressible finite hyperelasticity. Comput. Math. Appl. 72, 25-47 (2016)

29. Zdunek, A., Rachowicz, W.: A 3-field formulation for strongly transversely isotropic compressible finite hyperelasticity. Comput. Methods Appl. Mech. Eng. 315, 478-500 (2017)

30. Zdunek, A., Rachowicz, W.: A mixed higher order fem for fully coupled compressible transversely isotropic finite hyperelasticity. Comput. Math. Appl. 74, 1727-1750 (2017)

31. Zdunek, A., Rachowicz, W.: A mixed finite element formulation for compressible finite hyperelasticity with two fibre family reinforcement. Comput. Methods Appl. Mech. Eng. 345, 233-262 (2019)

32. Oden, J.T., Demkowicz, L.F.: Applied Functional Analysis. CRC Press, Boca Raton (1996) 